\title{
Moore and Seiberg's Equations and 3D Topological Field Theory
}

\author{
P. Degiovanni *

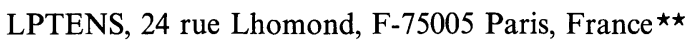

Received February 3, 1991; in revised form August 8, 1991

\begin{abstract}
We prove that any solution to Moore and Seiberg's equations defines a Projective Rational Topological Field Theory (PRTFT) using surgery presentations for decorated three-manifolds and Kirby's calculus.
\end{abstract}

\section{Table of Contents}

1. Position of the Problem . . . . . . . . . . . . . . . . . . . . . . 461

1.1. Notation and Results . . . . . . . . . . . . . . . . . . . . 461

1.2. Architecture of the Proof. . . . . . . . . . . . . . . . . . . . . 466

2. Building Blocks of RTFT in 3D . . . . . . . . . . . . . . . . . . . . . .467

2.1. Construction of the $H_{N} \mathrm{~s}$. . . . . . . . . . . . . . . . . . . . . . . . . 467

2.2. Graph Invariants in $S_{3} \ldots \ldots \ldots$. . . . . . . . . . . . . . . . . . . . 468

2.3. Partition Functions and Surgery Presentations. . . . . . . . . . . . . . . . 470

2.3.1. Surgeries . . . . . . . . . . . . . . . . . . . . . 470

2.3.2. Partition Function of a Surgery Presentation. . . . . . . . . . . . . 471

3. Topological Invariance from Kirby's Calculus . . . . . . . . . . . . . . . . . . .471

3.1. Equivalences Between Surgery Presentations. . . . . . . . . . . . . . . . . . 471

3.2. Topological Invariance of the Partition Function . . . . . . . . . . . . . . 475

4. Checking the Axioms of RTFTs . . . . . . . . . . . . . . . . . . . . . . . . . 478

4.1. Definition of Operators . . . . . . . . . . . . . . . . . . . 478

4.2. Unitarity Axioms . . . . . . . . . . . . . . . . . . . . . . . . . . . . . .480

4.3. Projective Functoriality in any 3-Manifold . . . . . . . . . . . . . . . . . 480

4.3.1. Surgery and Boundaries . . . . . . . . . . . . . . . . . . . . . . . 481

4.3.2. Clever Surgery Presentations . . . . . . . . . . . . . . . . . . . . . 481

4.3.3. Proof of Functoriality . . . . . . . . . . . . . . . . . . . . . .482

* After 1st October 1991: Laboratoire de Physique Théorique ENSLAPP de l'Ecole Normale Supérieure de Lyon, 46 Avenue d'Italie, F-69007 Lyon, France

$\star \star$ Unité Propre de Recherche du Centre National de la Recherche Scientifique, associée à l'Ecole Normale Supérieure et à l'Université de Paris-Sud 
4.4. Consequences of Functoriality . . . . . . . . . . . . . . . . . . . . . 483

4.4.1. Morse Theory Computations . . . . . . . . . . . . . . . . . . . . . . 483

4.4.2. About Projectivity. . . . . . . . . . . . . . . . . . . . . . . . . . . 487

4.4.3. Gluing Axiom. . . . . . . . . . . . . . . . . . . . . . . . . . . . . . . . . . . . 4887

A. Technicalities Around $F$ Matrices . . . . . . . . . . . . . . . . . . . . . . . . 490

A.1. Tetrahedral Symmetry of the $F$ Matrix . . . . . . . . . . . . . . . . . . . . 490

A.2. Unitarity of $F$ and $B$ Matrices. . . . . . . . . . . . . . . . . . . . . . . . . . . . . . . . . . 491

B. Graph Invariants in $S_{3} \ldots \ldots \ldots$. . . . . . . . . . . . . . . . . . . . . . . . . 492

B.1. Topological Invariance in $S_{3} \ldots \ldots$. . . . . . . . . . . . . . . 492

B.2. Properties of these Invariants . . . . . . . . . . . . . . . . . . . . . . . 496

B.2.1. Changing the Orientation of the Graph . . . . . . . . . . . . . . . . 496

B.2.2. Functoriality along $S_{2} \ldots \ldots \ldots$. . . . . . . . . . . . . . . . . . . . . 496

B.2.3. Other Bases of $H_{0, n}$. . . . . . . . . . . . . . . . . . . . . . . . . . . . . . 497

B.2.4. Partial Projective Functoriality for $g \geqq 1 \ldots$. . . . . . . . . . . . . . . 498

B.2.5. Miscalena . . . . . . . . . . . . . . . . . . . . . . . . . 503

\section{Introduction}

In 1986, Friedan and Shenker cast the idea that in RCFT, a finite dimensional Hilbert space was associated with every orientable surface possibly with punctures [1]. This space was nothing but the space of conformal blocks on this surface. They are holomorphic functions on some Teichmüller space and the space of conformal blocks is nothing but the space of holomorphic sections of a finite dimensional bundle over moduli space of Riemann surfaces of genus $g$ with $n$ punctures. Moreover, this bundle (Friedan-Shenker's bundle) is flat ${ }^{1}$ with respect to the connection defined by the energy-momentum tensor of the RCFT. One can classify projectively flat vector bundles over the moduli space by considering representations of the modular group $M^{*}(g, n)$ [2]. In RCFT, this representation arises through the monodromies of conformal blocks around non-trivial cycles in the moduli space ${ }^{2}$. Moore and Seiberg have discussed precisely these representations and they have found that a finite number of finite dimensional matrices entirely defined all possible monodromies of any conformal block on any Riemann surface. A complete set of relations (Moore and Seiberg's equations) ensures the modular covariance $^{3}$ and duality in any genus and with any number of punctures. However, these spaces remained quite mysterious and the lack of understanding them prevented any attempt at classifying these structures.

Because of these problems, it became quite urgent to find an interpretation of these data. A clever observation by Witten [3] in 1988 made it possible to interpret these spaces as the Hilbert spaces of states of a 3D field theory. In the case of WZW models associated with a simply connected compact Lie group, the associated 3D theory is the Chern-Simons theory associated with the same group. This mapping has been more precise by many authors among which [4-7] and it suggests a much more general pattern as was noticed in [3]. For instance, it became clear that Moore and Seiberg's equations could be obtained from the requirement of topological invariance [3, 8]: any RTFT defines a solution to Moore and Seiberg's equations. Henceforth, it is tempting to assume that we should be able to reconstruct the whole TFT from a solution to Moore and Seiberg's equations.

\footnotetext{
${ }^{1}$ Indeed, it is only a projectively flat bundle, and therefore all the representations of modular groups we shall consider in this paper are projective

${ }_{2}$ Thinking of sections of a bundle as multivalued functions

${ }^{3}$ Id est, that all defining relations of the modular group are satisfied
} 
The reconstruction of a 3D RTFT from a solution to Moore and Seiberg's equations is the purpose of this paper. More precisely, we shall reconstruct the algebraic structure of a RTFT. These algebraic aspects à la Segal are recalled in Sect. 1. In this first section, we also recall some basic facts about Moore and Seiberg's equations and present the plan of our reconstruction procedure. The following sections are devoted to this reconstruction. In Sect. 2, we define the Hilbert spaces of states and indicate how to define partition functions of boundaryless decorated manifolds using surgery presentations. Section 3 explains how to prove the topological invariance of these amplitudes using Kirby's calculus. Then, we will check in Sect. 4 that all axioms of PRTFT are satisfied and indicate how to compute amplitudes of manifold with boundaries using Morse theory. Finally, all technicalities concerning graph invariants in $S_{3}$ have been collected in Appendix B.

The interested reader should also read independent works on this matter. Topological invariants were defined by Kontsevitch in the case of undecorated closed manifolds [9] and also by Crane using Heegaard decompositions. Reshetikhin and Turaev [10] defined RTFT using Kirby's calculus and Quantum group which is an example of a modular Hopf algebra the representation theory of which provides us with a solution to Moore and Seiberg's equations. Other references have recently been brought to my attention at the "Rencontres Mathématiques de l'ENS" in Lyon: [11,12] which seem to share the same spirit as [10].

\section{Position of the Problem}

\subsection{Notation and Results}

Before explaining our method and the proof, let us recall briefly some notation. We shall also state precisely our final result.

We start from Moore and Seiberg's equations which arise in the context of Rational Conformal Field Theories (RCFTs). In this paper, we shall omit this aspect and we refer the reader to the original articles by Moore and Seiberg $[13,14,8]$, the related work of Fröhlich and his collaborators $[15,4]$ and papers by Rehren and Schröer [16]. Here, we shall consider these equations in a rather abstract manner. Several reasons led to this point of view: first of all, it is not clear at all that any solution to these equations defines a RCFT; secondly, they do not determine uniquely a RCFT ${ }^{4}$.

Let us recall some notation. We are given a finite set of indices $i$ called the line indices among which is $0 . i \rightarrow \hat{\imath}$ is a given involutive permutation of these indices and $\hat{0}=0$. Together with these line indices, we have some vertex indices $a$. For $i, j, k$ fixed, we have $N_{i, j, k}$ possible vertex indices defining a set $[i, j, k]$. These numbers are called the "fusion rules" and verify:

$$
N_{i, j, k} \text { is symmetric, } \quad N_{i, j, 0}=\delta_{i, \hat{\jmath}} \text {. }
$$

The involution is also defined on these indices and sends $a \in[i, j, k]$ on $\hat{a} \in[\hat{\imath}, \hat{\jmath}, \hat{k}]$. It implies $N_{\hat{\imath}, \hat{j}, \hat{k}}=N_{i, j, k}$. The image of a line or a vertex index by this involution is called the conjugated index. A vertex index of $[i, j, k]$ is conveniently

\footnotetext{
${ }^{4}$ As one easily sees by tensoring with the Moonshine module
} 
represented as a labelled trivalent vertex with oriented ordered incoming external lines $i, j$, and $k$. Indices can be used to color trivalent graphs.

Definition 1. A coloring of a trivalent graph is an assignment of a line (respectively vertex) index to each line (respectively vertex) of the graph together with an orientation of the lines. Reversing the orientation and turning each index into its conjugate is considered to give the same coloring. To any vertex with incoming lines $i, j$, and $k$, we assign a vertex index $a \in[i, j, k]$.

We also define the opposite coloring obtained by turning all indices into their conjugates. If $\mathscr{C}$ is a coloring of a given graph, the opposite coloring will be denoted by $\hat{\mathscr{C}}$.

Moore and Seiberg's data consist into a finite set of finite dimensional matrices. These are the duality matrices $F$ and $B( \pm)$ and the $g=1$ modular transformation matrices $S(j)$ and $T$. In the context of RCFT, they represent various monodromies of the conformal blocks understood as multivalued functions defined on some moduli space. The $F$ and $B$ matrices mix the colorings of a genus zero graph with four given external legs as indicated in Subsect. 2.1.

Let us now define the $S$ and $T$ matrices. Their matrix elements mix colorings of a one loop diagram with one external leg. We have:

$$
S_{(j, i, a)}^{\left(j^{\prime}, i^{\prime}, a^{\prime}\right)}=S(j)_{(i, a)}^{\left(i^{\prime}, a^{\prime}\right)} \delta_{j, j^{\prime}}, \quad T_{(j, i, a)}^{\left(j^{\prime}, i^{\prime}, a^{\prime}\right)}=e^{2 \pi i\left(h_{i}-c / 24\right)} \delta_{i, i^{\prime}} \delta_{j, j^{\prime}} \delta_{a, a^{\prime}} .
$$

Here, $j$ is the external line index, $i$ is the loop index and $a$ is the vertex index (idem for $j^{\prime}, i^{\prime}$, and $a^{\prime}$ ). We shall denote $S(0)$ by $S$ and $T(0)$ by $T$. Of course, $h_{i}-c / 24$ is only defined up to an integer. Our notation is reminiscent from RCFT where $h_{i}$ is the conformal dimension of a primary field and $c$ is the central charge of the theory. As we shall see in the next paragraph, it is indeed necessary to fix $h_{i}$ modulo 2 : the $B( \pm)$ matrices represent half-monodromies of the fields on the plane and therefore, they produce squareroots of the phases $\exp \left(2 \pi i h_{i}\right)$ for the twisted lines. We impose that $h_{0} \equiv 0(\bmod 2) . F$ and $S$ are supposed to be invertible. We are now ready to write down Moore and Seiberg's equations.

We shall introduce here the $\Omega( \pm)$ and $\Theta( \pm)$ matrices which are some particular cases of $B( \pm)$. Our notation follows Moore and Seiberg's one. They define $\Omega$ and $\Theta$ in terms of $B$ and isomorphisms $\sigma_{12}: \operatorname{Vect}([i, j, k]) \rightarrow \operatorname{Vect}([j, i, k])$ (or any other permutation of the indices). We refer the reader to their work for more details. $\Omega( \pm)$ and $\Theta( \pm)$ can be computed using the conformal dimensions and $F, B, S$, and $T$ verify a finite number of polynomial equations:

- $\Omega$ and $\Theta$ matrices act on each $\operatorname{Vect}([i, j, k])$ separately and each component is denoted $\Omega_{i j k}( \pm)\left(\right.$ respectively $\left.\Theta_{i j k}( \pm)^{5}\right)$ :

$$
\begin{aligned}
& \Omega( \pm)_{a, a^{\prime}}=\exp \left( \pm \pi i\left(h_{k}+h_{j}-h_{i}\right)\right) \delta_{a^{\prime}, \sigma_{12}(a)}, \\
& \Theta( \pm)_{a, a^{\prime}}=\exp \left( \pm \pi i\left(h_{i}+h_{k}-h_{j}\right)\right) \delta_{a^{\prime}, \sigma_{13}(a)} .
\end{aligned}
$$

- Genus zero equations:

$$
\begin{gathered}
F_{23} F_{12} F_{23}=P_{23} F_{13} F_{12}, \\
F(\Omega( \pm) \otimes \mathbf{1}) F=(\mathbf{1} \otimes \Omega( \pm)) F(\mathbf{1} \otimes \Omega( \pm)), \\
B( \pm)=(\Omega(\mp) \otimes \mathbf{1}) F(\mathbf{1} \otimes \Omega( \pm)) .
\end{gathered}
$$

5 The careful reader will have noticed that some signs can appear in $\Omega$ and $\Theta$ due to the symmetry or antisymmetry of the vertices understood as intertwiners between representations of the chiral algebra (in the context of RCFT). For the sake of simplicity, we shall not mention them in the rest of this work 
- Genus one equations:

$$
\begin{gathered}
(S(j) T)^{3}=\oplus_{i}\left(\Theta_{i j i}(-) \circ P_{\left.\left[\begin{array}{c}
\hat{i} \\
j, i
\end{array}\right]\right),}\right. \\
(S(j))^{2}=\oplus_{i}\left(\Theta_{\hat{i} j i}(-) \circ P\left[\begin{array}{c}
\hat{i} \\
j, i
\end{array}\right]\right), \\
(S \otimes \mathbf{1}) F(\mathbf{1} \otimes \Theta(-) \Theta(+)) F^{-1}\left(S^{-1} \otimes \mathbf{1}\right)=F P F^{-1}(1 \otimes \Omega(-)) .
\end{gathered}
$$

Here, $P\left[\begin{array}{c}\hat{i}, i \\ j, i\end{array}\right]$ denotes the projector on the space of colorings with loop index $i$ and external line index $j$. We need some extra hypotheses:

- The following "reality conditions" hold:

$$
h_{\hat{k}}=h_{k}, \quad F_{\hat{p}, \hat{q}}\left[\begin{array}{ll}
\hat{c} & \hat{d} \\
\hat{a} & \hat{b}
\end{array}\right]=\left(F_{p, q}\left[\begin{array}{ll}
c & d \\
a & b
\end{array}\right]\right)^{*} .
$$

- Fusing matrices acting on a block with an external line set to 0 are trivial. In particular if $t_{i}$ denotes the unique ${ }^{6}$ coupling in [iî0]:

$$
F_{i, \hat{k}}\left[\begin{array}{cc}
t_{k} & b \\
t_{i} & a
\end{array}\right]=\delta_{a, \sigma_{123}(b)} .
$$

- For all line indices $i, S_{0}^{i}>0$.

- Here is a technical hypothesis on $F$ matrices:

$$
F_{p, 0}\left[\begin{array}{cc}
t_{i} & t_{j} \\
a & b
\end{array}\right]=0 \quad \text { if } \quad a \neq \hat{b} .
$$

These properties will be used in Appendix A to show some properties of $F$ from the equations. More precisely, we shall see that in a special gauge, the $F$ matrix has the so-called tetrahedral symmetry [17]. Note that:

Warning. In the rest of this paper, we shall assume that such a gauge choice has been performed!

These data appear in Moore and Seiberg's review in a slightly different form. They use the concept of a modular tensor category which was introduced by Frenkel [18] and is believed to be an appropriate generalization of the Tannakian category concept. However, we prefer to use the above presentation and we shall speak of a solution to Moore and Seiberg's equations.

On the other hand, we define what we mean by tridimensional rational topological field theory. This axiomatics has been developed originally by Segal [19] in the context of CFT, [7, 20-23] in the case of TFTs. Let us recall the definition of a TFT in $d$ dimensions from this point of view.

We start from a category Ma the objects of which are $d-1$ dimensional oriented manifolds. We define $\hat{N}$ by $\partial(N \times[0,1])=N \cup \hat{N}$, it has reversed orientation. If $N$ is an object, $\hat{N}$ is also an object and $\emptyset$ is an object. Morphisms are $d$-dimensional parametrized cobordisms. An element of $\operatorname{hom}_{\mathbf{M a}}\left(N_{1}, N_{2}\right)$ is a $d$-dimensional manifold with boundary $N_{1} \cup \hat{N}_{2}$ together with $f_{1,2}$ parametrizations of $N_{1}$ and $N_{2}$ as submanifolds of $M$. Let $M$ and $M^{\prime}$ be two manifolds with parametrized boundaries, we suppose that $N \subset \partial M$ and $N \subset \partial M^{\prime} . \varphi: \partial M \mapsto N$ and $\varphi^{\prime}$ denote the parametrizations of the boundaries. Then $M \#_{g} M^{\prime}$ will denote the

\footnotetext{
${ }^{6}$ We shall be sloppy about the order of external lines in this case
} 
manifold obtained by gluing $M$ and $M^{\prime}$ via $g \in \operatorname{Diff}_{+}(N)$ : we identify $P \in \partial M$ with $P^{\prime} \in \partial M^{\prime}$ if and only if $(g \circ \varphi)(P)=\varphi^{\prime}\left(P^{\prime}\right)$. If $g=\mathbf{1}_{N}$, we shall omit it in $\#_{g}$. This defines our composition law \# on morphisms. Finally, if $M$ is a manifold such that two copies of $N$ are present in its boundary, then $M_{[N]}$ denotes the manifold obtained by gluing these copies together, this supresses $N \cup \hat{N}$ of $M$ 's boundary. If $M$ is a morphism, $\hat{M}$ and $M_{[N]}$ are also morphisms. If $N$ and $N^{\prime}$ are two disjoint objects, $N \cup N^{\prime}$ is supposed to be an object. This property is also assumed for morphisms. Finally, $N \times[0,1]$ parametrized trivially at its boundary is a unit associated with the object $N$.

Finally, we can consider morphisms as isomorphic:

Definition 2. Let $M$ and $M^{\prime}$ be two three-manifolds with boundaries isomorphic to $N$. An orientation preserving diffeomorphism $f \in \operatorname{Diff}_{+}\left(M, M^{\prime}\right)$ is an isomorphism from $M$ onto $M^{\prime}$ if and only if $\left.\varphi^{\prime} \circ f\right|_{\partial M}=\varphi$ where $\left.f\right|_{\partial M}$ denotes the restriction of $f$ to $M^{\prime}$ 's boundary and $\varphi: \partial M \rightarrow N$ and $\varphi^{\prime}: \partial M^{\prime} \rightarrow N$ are the parametrizations of $M$ and $M^{\prime} s$ boundaries.

As we know from various papers [17], we shall consider decorated manifolds, that is to say manifolds equipped with a decorating submanifold. We impose that isomorphisms be compatible with this decoration: it must send one decoration onto the other. In the three-dimensional case, decorations are $\phi^{3}$ colored and framed graphs. Henceforth, an isomorphism has to respect colorings and framings of these graphs. Following Reshetikhin and Turaev [24], we shall consider each line as a small ribbon, which defines its framing. In our discussion, Ma's objects are closed orientable surfaces with marked points and a non-zero tangent vector at each point. Morphisms are interpolating three-manifolds, decorated by a trivalent framed diagram interpolating between marked points. When intersecting the boundary of a three-manifold, a ribbon defines a non-zero tangent vector.

On the other hand, an algebraic category $\mathbf{S p}$ is given. Its objects are Hilbert spaces and $\mathbf{C}$ is one of the objects. Obviously, hom Sp $_{\text {p }}\left(H, H^{\prime}\right) \subset \mathscr{L}\left(H, H^{\prime}\right)$ and the composition law is the ordinary product of linear operators. We assume stability under tensor product for objects as well as morphisms. If $H$ is an object and $H^{*}$ its dual, it is also an object. Similarily, if $u \in \operatorname{hom}_{\mathbf{s p}}\left(H, H^{\prime}\right)$, then $u^{\dagger} \in \operatorname{hom}_{\mathbf{S p}}\left(H^{\prime}, H\right)$ and we also suppose that $\mathbf{1}_{\boldsymbol{H}}$ is the unit associated with $H$.

This enables us to define a TFT based on these categories:

Definition 3. $A$ Topological Field Theory is a contravariant functor $\Phi$ between Ma and Sp such that, if for any object $N$ and any morphism $M$ of the first category $\Phi[N]$ is denoted by $H_{N}$ and $\Phi[M]$ is denoted by $\phi[M]$, the following properties hold:

$$
\begin{gathered}
\phi\left[M_{[Y]}\right]=\operatorname{Tr}_{H_{Y}}(\phi[M]), \\
H_{\hat{N}}=\left(H_{N}\right)^{*}, \quad \phi[\hat{M}]=(\phi[M])^{\dagger}, \\
H_{N \cup N^{\prime}}=H_{N} \otimes H_{N^{\prime}}, \quad \phi\left[M \cup M^{\prime}\right]=\phi[M] \otimes \phi\left[M^{\prime}\right], \\
H_{\emptyset}=\mathbf{C}, \quad \phi[N \times[0,1]]=\mathbf{1}_{H_{N}} .
\end{gathered}
$$

Moreover, $H_{N}$ only depends on the topology of $N$ and if $M$ and $M^{\prime}$ are isomorphic, $\phi[M]=\phi\left[M^{\prime}\right]$.

The theory is called rational when all Hilbert spaces are finite dimensional and in this paper, only Rational TFT (RTFT) are considered. When $M$ is a 
boundaryless manifold, the associated operator is a number called the partition function denoted by $Z[M]$.

Closely related to this arises the notion of a projective topological field theory (PTFT):

Definition 4. A Projective Topological Field Theory is a correspondance $\Phi$ between Ma and $\mathrm{Sp}$ which associates $H_{N}$ with every object $N$ of $\mathrm{Ma}$ and $\phi[M] \in \operatorname{hom}_{\mathbf{S p}}\left(H_{A}, H_{B}\right)$ with every $M \in \operatorname{hom}_{\mathbf{M a}}(A, B)$, such that:

$$
\begin{gathered}
H_{\hat{N}}=\left(H_{N}\right)^{*}, \quad \phi[\hat{M}]=\phi[M]^{\dagger}, \\
H_{N \cup N^{\prime}}=H_{N} \otimes H_{N^{\prime}}, \quad \phi\left[M \cup M^{\prime}\right]=\phi[M] \otimes \phi\left[M^{\prime}\right], \\
H_{\emptyset}=\mathbf{C}, \quad \phi[N \times[0,1]] \propto \mathbf{1}_{H_{N}},
\end{gathered}
$$

and $H_{N}$ only depends on N's topology and for $M$ and $M^{\prime}$ isomorphic, $\phi[M]=\phi\left[M^{\prime}\right]$. Moreover, for each pair of composable morphisms $\left(M_{1}, M_{2}\right)$, there exists $\mu\left(M_{1}, M_{2}\right) \in \mathbf{C}$ satisfying:

$$
\phi\left[M_{1} \# M_{2}\right]=\mu\left(M_{1}, M_{2}\right) \times \phi\left[M_{2}\right] \circ \phi\left[M_{1}\right]
$$

with the following compatibility condition:

$$
\mu\left(M_{1} \# M_{2}, M_{3}\right) \mu\left(M_{1}, M_{2}\right)=\mu\left(M_{1}, M_{2} \# M_{3}\right) \mu\left(M_{2}, M_{3}\right) .
$$

Equation (21) is called "projective functoriality" and $\mu$ should be called a cocycle. Axioms of a PTFT imply that $\mu$ satisfies the following equations:

$$
\begin{aligned}
\mu\left(M_{1} \cup M_{1}^{\prime}, M_{2} \cup M_{2}^{\prime}\right) & =\mu\left(M_{1}, M_{2}\right) \mu\left(M_{1}^{\prime}, M_{2}^{\prime}\right), \\
\mu\left(M_{1}, M_{2}\right)^{*} & =\mu\left(\hat{M}_{2}, \hat{M}_{1}\right) .
\end{aligned}
$$

It can easily be proved that a PTFT can be turned into a true TFT defined on an extension $\mathbf{M a}_{e}$ of $\mathbf{M a}$ by $\mathbf{C}$. I recall that a central extension of a category $\mathbf{X}$ by $\mathbf{C}^{7}$ has the same objects than $\mathbf{X}$ and for any morphism $f$ of $\mathbf{X}$ we are given $L_{f} \simeq \mathbf{C}$ and, for each pair of composable morphisms $(f, g), \mu(f, g) \in \operatorname{hom}\left(L_{f} \times L_{g}, L_{f g}\right)$ such that:

$$
\mu(f, g h) \circ\left(\mathbf{1}_{L_{f}} \otimes \mu(g, h)\right)=\mu(f g, h) \circ\left(\mu(f, g) \otimes \mathbf{1}_{L_{h}}\right) .
$$

In the extended category, morphisms from $A$ to $B$ are pairs $(f, \lambda)$, where $\lambda \in L_{f}$ and $f \in \operatorname{hom}_{\mathbf{x}}(A, B)$. Composition of morphisms is defined by:

$$
(f, \lambda) \circ\left(g, \lambda^{\prime}\right)=\left(f g,\left(\mu(f, g)\left(\lambda, \lambda^{\prime}\right)\right)^{-1}\right) .
$$

Our aim is to clarify the relationship between Moore and Seiberg's equations and $3 \mathrm{D}$ topology. We want to prove that these equations are indeed the "equations of motion" of a 3D PRTFT. Indeed, we shall prove the following result:

Theorem 1. Any solution to Moore and Seiberg's equations defines a Projective Rational Topological Field Theory in 3 dimensions through the procedure described by Witten in [3] and [17].

\footnotetext{
7 Or more generally by any abelian group ...
} 
We shall come back later to the interpretation of the cocycle. Let us recall that a TFT defines a true representation of modular groups of $d-1$-manifolds whereas a PTFT defines a projective representation of these groups. Interpreting geometrically the cocycle $\mu\left(M_{1}, M_{2}\right)$ should provide us with a better understanding of central extensions of all modular groups, in any topology.

\subsection{Architecture of the Proof}

Let us now describe the structure of our proof. All technical details will be reported in the next sections of this paper and we shall only describe the main steps in this part.

Our idea is to define a topological theory by matrix elements associated with an arbitrary three-manifold. Our starting data will be a solution to Moore and Seiberg's equations as described in the preceding subsection. We shall firstly define the Hilbert space associated with a surface of genus $g$ and $n$ punctures by describing a basis of this space in Sect. 2.1.

The next step is to define matrix elements of the operator associated with any three-manifold. The simplest case is provided by manifolds without boundary. We want to associate with them a number.

In the case of the three-sphere with a trivalent framed graph $K$ in it, we shall use a transfer matrix method. We decompose the three-sphere by cutting it along two-spheres. Between two cuts lies a vertex or a pair creation or pair annihilation. With any such event, we associate a matrix acting on the appropriate $H_{0, n} \mathrm{~s}$ and the partition function is defined as the product of these matrices. Our procedure defines a topological invariant for framed trivalent graphs in $S_{3}$. Such a statement has been proved by Fröhlich [4] and in the context of quantum groups, by Reshetikhin and Turaev [24]. We recall how it works in Appendix B.

In the case of a three-manifold with no boundary and different from $S_{3}$, we use a surgery presentation of $M$ to go back to the case of a three-sphere [25]. Kirby [26] and Fenn and Rourke [27] have described how two surgery presentations of a given three-manifold are related. We adapt it to the case of a three-manifold with a framed trivalent graph embedded in it. We define the partition function of a surgery presentation and prove that it only depends on the underlying decorated three-manifold in Sect. 3.

To define the matrix element of $\phi[M]$ between two vectors of bases defined in Sect. 2.1, we consider the partition function associated with the closed manifold obtained by saturating each connected component of $M$ 's boundary with the handlebody associated with the vector we use.

As we shall see, the only non-trivial property is the projective functoriality of our correspondence between manifolds and operators.

To prove this statement, we shall proceed in several steps. In Lemma 9, we show that partial projective functoriality in $S_{3}$ implies projective functoriality for any three-manifold with no boundary and therefore for any three-manifold. In the case of a splitting of $S_{3}$ by a trivially embedded surface ${ }^{8}$, Eq. (21) is proved by a detailed computation of both sides using only functoriality along a two-sphere. The latter property is known to be true in $S_{3}$.

Finally, property (21) enables us to make contact with the generalized transfer matrix method. This method is based on the use of Morse functions. Using this

8 That is to say, splitting $S_{3}$ into two handlebodies 
technique, one can show that the gluing axiom is satisfied (see Proposition 4.4.3) for the RTFT defined on the extended category $\mathbf{M a} \mathbf{a}_{e}$.

\section{Building Blocks of RTFT in 3D}

In this section, we shall describe how to define precisely the Hilbert spaces of the 3D theory and the amplitudes.

\subsection{Construction of the $H_{N}$ S}

We shall use these spaces in Sect. 4.1 to define operators associated with manifolds with boundary. Let us consider $N$ a two dimensional manifold without boundary. For the sake of simplicity, we shall firstly assume that $N$ is connected. More precisely, $N$ is of genus $g$ and has $n$ marked points. We consider then a trivalent graph $\mathscr{G}$ of genus $g$ and with $n$ external legs, with fixed framing, embedded as a 1-skeleton of the genus $g$ handlebody. Then we define $H_{N}$ as follows:

Definition 5. A basis of $H_{N}$ is indexed by the set of colorings of $\mathscr{G}$. We shall note $\left|T_{g}, \mathscr{G}_{\mathscr{C}}\right\rangle$ the vector corresponding to the colouring $\mathscr{C}$. We define $H_{\emptyset}=\mathbf{C}$.

We shall consider that these vectors are associated with genus $g$ handlebodies with $\mathscr{G}_{C}$ embedded in it. This justifies our notation: $\left|T_{g}, \mathscr{G}_{\mathscr{C}}\right\rangle$. When $N$ is of genus $g$ with $n$ punctures, we shall note $H_{N}$ as $H_{g, n}$. The scalar product on this space is defined following Witten [17].

$$
Q\left(\left|T_{g}, \mathscr{G}_{\mathscr{C}^{\prime}}\right\rangle,\left|T_{g}, \mathscr{G}_{\mathscr{C}}\right\rangle\right)=\left\langle T_{g}, \mathscr{G}_{\mathscr{C}^{\prime}} \mid T_{g}, \mathscr{G}_{\mathscr{C}}\right\rangle=\delta_{\left[\mathscr{C}^{\prime},\left[\mathscr{C}^{\prime}\right]\right.} \frac{\sqrt{S_{0}^{l_{1}} \ldots S_{0}^{l_{n}}}}{\left(S_{0}^{0}\right)^{g-1+n / 2}},
$$

where $S$ is the matrix appearing in Moore and Seiberg's data and $\delta_{[\mathscr{C}],\left[\mathscr{C}^{\prime}\right]}$ is a delta function on the set of colourings of $\mathscr{G} . l_{1}, \ldots, l_{n}$ are the indices of $\mathscr{G}$ 's external legs. $\left(\left|T_{g}, \mathscr{G}_{\mathscr{C}}\right\rangle\right)_{\mathscr{C}}$ is an orthogonal basis of $H_{N}$.

In the case where $N$ is not connected, let $N=\bigcup_{i \in I} N_{i}$ be the decomposition of $N$ into connected components. We define $H_{N}$ to be $\bigotimes_{i \in I} H_{N_{i}}$. This ensures that $H_{N \cup N^{\prime}}$ $=H_{N} \otimes H_{N^{\prime}}$. Moreover, $H_{\hat{N}}=\left(H_{N}\right)^{*}$ can be imposed without any problem. Notice that $H_{N}$ only depends on $N$ 's topology.

We can change the basis by changing $\mathscr{G}$ into $\mathscr{G}^{\prime}$. Any two such graphs are related by elementary moves $F$ and $B$. Therefore, we define the change of bases associated with such elementary moves by:

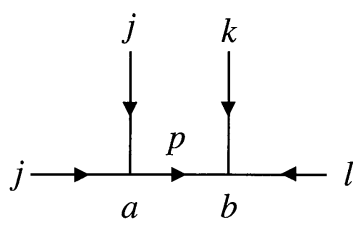

$$
=\sum_{c, d} F_{p, q}\left[\begin{array}{ll}
c & d \\
a & b
\end{array}\right]
$$
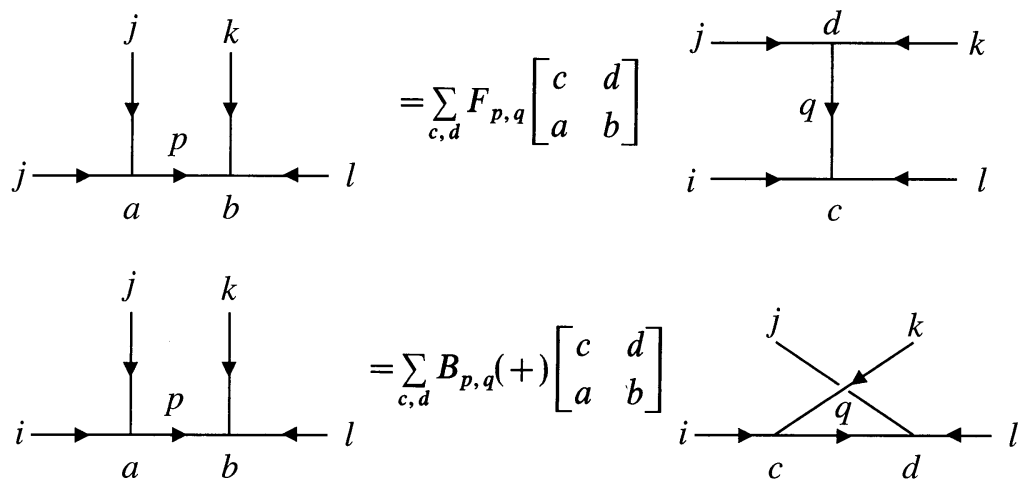


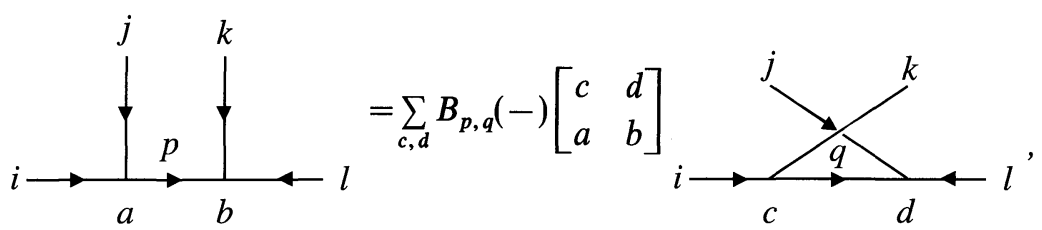

where we have represented the part of the graphs that has been affected by the moves. For a more general move, we decompose it into elementary moves and take the product of the corresponding matrices. As proved in [14, Appendix B], the result does not depend on the decomposition we chose. Henceforth we can write:

$$
\left|T_{g}, \mathscr{G}_{\mathscr{C}}\right\rangle=\sum_{\mathscr{\mathscr { G } ^ { \prime }}} P\left(\mathscr{G}_{,}, \mathscr{G}^{\prime}\right)_{\mathscr{C}, \mathscr{C}^{\prime}}\left|T_{g}, \mathscr{G}_{\mathscr{C}^{\prime}}^{\prime}\right\rangle
$$

Note that the braiding and fusing matrices are unitary matrices, as checked in Appendix A. Henceforth, the normalization of scalar products is the same for all $\mathscr{G}$ at given genus and number of punctures.

All this defines a set of vector spaces which verify all properties needed to be the objects of Sp.

\subsection{Graph Invariants in $S_{3}$}

Let us define the partition function of a framed $\phi^{3}$ diagram $K$ embedded in the sphere $S_{3}$ following [17]. We can consider that $K \subset \mathbf{R}^{3} C S_{3}$ by choosing a particular $\mathbf{R}^{3}$ in $S_{3}$. We then project on a plane and assume the projection to be regular: nothing but crossings and vertices appear on the projection. Then, we choose a Morse function on the three-sphere which separates these events (see Fig. 1). $S_{3}$ is sliced into two three-balls $B_{3}$ between which lie several decorated cylinders $S_{2} \times[0,1]$. Let us denote them by $\left(C_{k}\right)_{k \in\langle 1, n\rangle}$. Any of these contains one event of the following type ${ }^{9}$ :
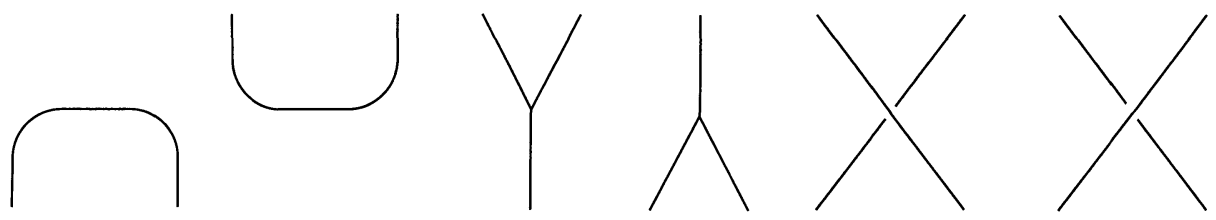

that is to say, pair annihilations and creations, vertices and braidings $\left(C_{-}\right.$and $C_{+}$ respectively). Let us recall which operators correspond to these events. In the following list, we suppose that the framing is normal. That is to say defined by considering lines as ribbons pointing towards the reader ${ }^{10}$. The operator corresponding to a decorated cylinder $C$ will be denoted by $\phi[C]: H_{0, n} \rightarrow H_{0, m}$.

9 Arrows are omitted

10 Henceforth this notion is not intrinsic in opposition to the zero framing of a knot

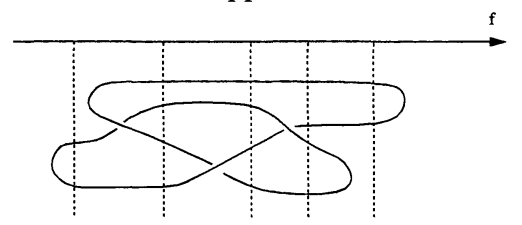

Fig. 1. A Morse function for the trefoil knot 
Vertices. In this case, one line split into two and it introduces the fusing matrix:

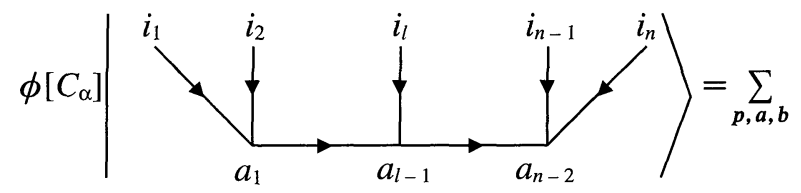

$F_{p, \hat{\imath}_{l}}\left[\begin{array}{cc}\hat{a}_{l-1} & \hat{Q} \\ \hat{b} & \hat{a}\end{array}\right]$

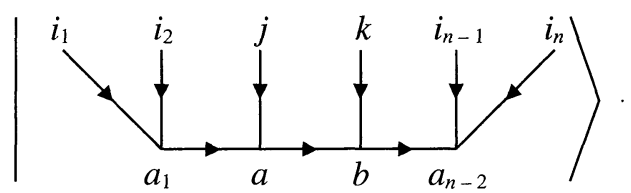

The vertex carries index $\alpha$ and line $i_{l}$ is split into lines with indices $j$ and $k$. We can also fuse two lines and it gives.

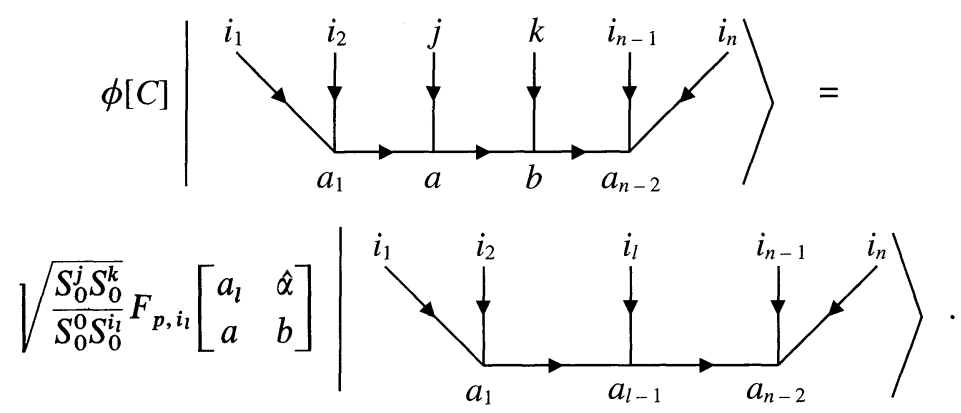

Pair Creation and Annihilation. These are defined by specializing $i_{l}=0$ in the above definitions.

Using our technical hypotheses [Eq. (11)] and tetrahedral symmetry (see Appendix A) the reader will easily find simple expressions for these events.

Crossings. Two possible crossings lead to two braiding matrices:

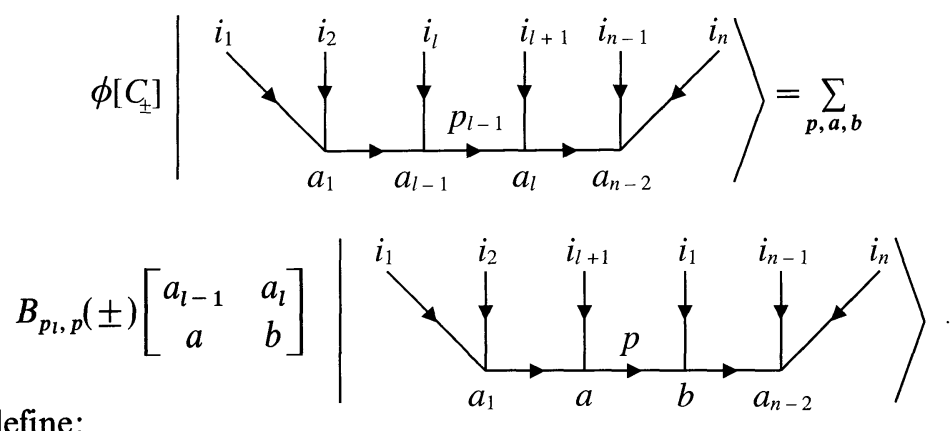

We define:

Definition 6. Let $K$ be a graph in $S_{3}$ with normal framing and:

$$
\left[S_{3}, K\right]=B_{3} \cup C_{1} \cup \ldots \cup C_{n} \cup B_{3}
$$

be a decomposition of $\left[S_{3}, K\right]$. The partition function associated with this decomposition is ${ }^{11}$ :

$$
S_{0}^{0} \prod_{k=1}^{n} \phi\left[C_{n-k}\right] \text {. }
$$

11 The $S_{0}^{0}$ is due to the two three-balls $B_{3}$ needed to close a cylinder into a sphere $S_{3}$ 
Let us now take the effects of the framing into account. We choose a decomposition of the three-sphere. For each line $l$ of the graph, the framing differs from the normal framing by $n_{l}$ units. Let $i_{l}$ be the index carried by line $l$. The effect of changing the framing by one unit of a line carrying index $j$ is to multiply the partition function by $\exp \left(2 \pi i h_{j}\right)$ :

$$
Z=Z_{\perp} \prod_{l \text { line }} \exp \left(2 \pi i n_{l} h_{i_{l}}\right),
$$

where $Z_{\perp}$ is computed using a normal framing.

We may wonder to what exent these numbers depend on the way we slice $S_{3}$. Using an adaptation of the proof of Reshetikhin and Turaev in the context of quantum groups [24], we explain in Appendix B how to prove the following result:

Lemma 1. The number defined by Definition 6 and Eq. (25) does not depend on the decomposition of $S_{3}$ we choose and therefore is a topological invariant for framed trivalent graphs in $S_{3}$.

Remark. We emphasize that operators $\phi[C]$ associated with decorated cylinders in this section coincides with the ones we shall define in Subsect. 4.1. Moreover, one can easily check that $\left\langle\mathscr{G}_{\mathscr{C}} \mid \mathscr{G}_{\mathscr{C}}\right\rangle=Z\left[S_{3}, \mathscr{G}_{\mathscr{C}} \# \mathscr{G}_{\mathscr{G}}\right]$.

\subsection{Partition Functions and Surgery Presentations}

In this section, we define a number associated with a surgery presentation of a decorated three-manifold.

2.3.1. Surgeries. Let us define precisely the surgery operation around a framed link $L$. The idea is to find tubular neighbourhoods of each component of the link, cut them away from our initial manifold and reglue them back in a non-trivial way. More precisely, a framed circle in $N$ defines an embedding $i$ of $D_{2} \times S_{1}$ in $N$. We note $C=i\left(\{0\} \times S_{1}\right)$. On $D_{2} \times S_{1}$, we have natural cycles $(a)_{0}$ and $(b)_{0}$. Before surgery, they are glued on $\left(a^{\prime}\right)$ and $\left(C^{\prime}\right)$ drawn on $\partial\left(N \backslash i\left(D_{2} \times S_{1}\right)\right)$. $\left(C^{\prime}\right)$ is a curve parallel to $(C)$ which defines its framing as in Fenn and Rourke's work. Surgery is defined by:

Definition 7. Performing a surgery on $N$ along $C$ consists into extracting $i\left(D_{2} \times S_{1}\right)$ and gluing it back with the identification:

$$
\begin{aligned}
& \left(a_{0}\right) \mapsto\left(C^{\prime}\right), \\
& \left(b_{0}\right) \mapsto-\left(a^{\prime}\right) .
\end{aligned}
$$

In the case of a link, we perform surgery along each component of the link.

In the case of the three-sphere, a $\operatorname{knot} C$ has a privileged framing associated with a Seifert surface. It is the $n=0$ framing. On $\partial\left(i\left(D_{2} \times S_{1}\right)\right)$, we draw the $(a)$ and $(b)$ cycles by reference to this privileged framing. The corresponding cycles on $\partial\left(N \backslash i\left(D_{2} \times S_{1}\right)\right)$ are called $\left(a^{\prime}\right)$ and $\left(b^{\prime}\right)$ as indicated on Fig. 2. If $n \in \mathbf{Z}$ denotes $C$ 's framing, identification after surgery is given by:

$$
\begin{aligned}
& (a) \rightarrow n\left(a^{\prime}\right)+\left(b^{\prime}\right), \\
& (b) \rightarrow-\left(a^{\prime}\right) .
\end{aligned}
$$




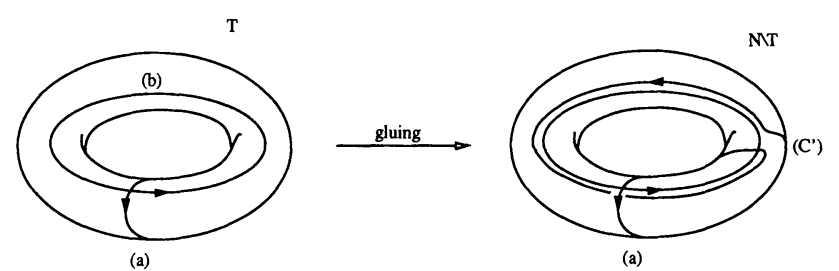

Fig. 2. The $(a)$ and $(b)$ cycles on $T_{i}$ and the corresponding $\left(a^{\prime}\right),\left(b^{\prime}\right)$ cycles on $\partial\left(N \backslash T_{i}\right)$. $\left(C^{\prime}\right)$ which defines an $n=-1$ framing of this unknotted circle is shown on this picture

Lickorish proved in [25] that any three-manifold without boundary can be obtained by surgery on $S_{3}$ along a framed link $L$. It is quite clear that starting from such a manifold $M$, equipped with a trivalent framed graph $K \subset M$, one can find $\bar{K}$ $C\left(S_{3} \backslash L\right)$ such that performing surgery on $\left[S_{3}, \bar{K}\right]$ along $L$ gives back $[M, K]$. By analogy with the preceding case, we say that $[L, \bar{K}]$ is a surgery presentation of $[M, K]$.

As explained by Kirby, there is a canonical way to associate a 4-dimensional manifold bounding $M$ with every surgery presentation $L$ of $M$. We start from $B_{4}$ the boundary of which is $S_{3}$, and given a tubular neighbourhood $T_{i}$ for each component of $L$, we glue $D_{2} \times D_{2}$ using $L$ 's framing. We define $\sigma(L)$ to be the signature of the intersection form on $\mathrm{H}_{2}\left(W_{L}, \mathbf{R}\right)$.

2.3.2. Partition Function of a Surgery Presentation. Let us now define the partition function of a surgery presentation $[L, \bar{K}]$ where $L$ is a framed link and $\bar{K}$ a framed coloured trivalent graph.

Definition 8. Let $L$ be a framed link and $\bar{K}$ a framed coloured trivalent graph in $S_{3}$, we define

$$
Z_{s}[L, \bar{K}]=e^{-2 \pi i c \sigma(L) / 8} \sum_{\mathscr{C}}\left[\prod_{k=1}^{n}\left(\left(e^{2 \pi i c / 24} T\right)^{n_{k}} S\right)_{\mathscr{C}_{k}}^{0}\right] Z\left[S_{3}, L_{\mathscr{C}}, \bar{K}\right]
$$

where the sum is over all colorings of $L$ and $L_{\mathscr{C}}$ has framing zero ${ }^{12}$.

This definition is extended to the case $L=\emptyset$ by $Z_{s}[\emptyset, \bar{K}]=Z\left[S_{3}, \bar{K}\right]$.

\section{Topological Invariance from Kirby's Calculus}

In order to prove that the number defined by Eq. (27) is indeed a topological invariant for decorated boundaryless manifolds, we shall determine when two surgery presentations $[L, \bar{K}]$ and $\left[L^{\prime}, \bar{K}^{\prime}\right]$ give rise to the same manifold. This is the object of Subsect. 3.1. Then, in Subsect. 3.2, we shall prove that the partition function $Z_{s}[L, \bar{K}]$ only depends on the result of the surgery.

\subsection{Equivalences Between Surgery Presentations}

Kirby [26] and Fenn and Rourke [27] gave equivalence criteria in the case of undecorated manifolds. We shall extend this to the case of decorated manifolds. The equivalence notion we shall use is quite natural and can be expressed as:

12 We could as well have left the initial framing but we decided to take it into account through $\left(T^{n_{k}}\right)_{\mathscr{C}_{k}}^{\mathscr{C}_{k}}$ for component $k$. This convention will hold all along this paper 
Definition 9. Two surgery presentations $[L, \bar{K}]$ and $\left[L^{\prime}, \bar{K}^{\prime}\right]$ are said to be equivalent if and only if the decorated manifolds $\left[M_{L}, K\right]\left(\left[M_{L^{\prime}}, K^{\prime}\right]\right.$ respectively) they give rise to are such that there exists $F \in \operatorname{Diff}\left(M_{L}, M_{L^{\prime}}\right)$ sending $K$ onto $K^{\prime}$ modulo an isotopy and preserving the colorings and framings of these graphs.

We note $[L, \bar{K}] \simeq\left[L^{\prime}, \bar{K}^{\prime}\right]$. In particular, this implies the weaker notion of equivalence used by Kirby:

Definition 10 (Kirby). Two framed links in $S_{3}$ are weakly equivalent if and only if the manifolds they produce by surgery are diffeomorphic: $M_{L} \simeq M_{L^{\prime}}$.

According to Kirby [26], the weak equivalence notion is tantamount to the existence of a finite sequence of moves starting at $L$ and ending at $L^{\prime}$ (Kirby's moves). In the case of a decorated manifold, we can describe the effect of these moves on $\bar{K} \subset S_{3}$. But this does not describe all $\left[L^{\prime}, \bar{K}^{\prime}\right]$ s equivalent to $[L, \bar{K}]$. For a fixed $L$ in $S_{3}$, many graphs $\bar{K}^{\prime}$ can be found with $\left[L, \bar{K}^{\prime}\right] \simeq[L, \bar{K}]$. We must describe them. Clearly, any $\left[L^{\prime}, \bar{K}^{\prime}\right] \simeq[L, \bar{K}]$ can be transformed into a $\left[L, \bar{K}^{\prime \prime}\right] \simeq[L, \bar{K}]$ through a finite number of Kirby's moves. Therefore, the knowledge of all $\overline{K^{\prime}}$ such that $\left[L, \bar{K}^{\prime}\right] \simeq[L, \bar{K}]$ solves the equivalence problem.

Kirby's Moves on $\phi^{3}$ Diagrams. First of all, let us recall what are Kirby's moves in the case where no trivalent diagram is present. We refer the reader to [26] and [27] for details. Given two circles in $S_{3}, l\left(C, C^{\prime}\right)$ denotes their linking number.

Definition 11. Let $C$ be an unknotted component of $L$ with framing $\varepsilon= \pm 1$, the $K$-move is defined by removing $C$ from $L$ and twisting the set of curves belonging to $L$ that pierce a disk with boundary $C$ as indicated on Fig. 3. Moreover, the framing $n_{i}$ of any component of $L$ is shifted into $n_{i}-\varepsilon l\left(C, C_{i}\right)^{2}$.

Kirby's basic result is:

Theorem 2 (Kirby). $L$ and $L^{\prime}$ are weakly equivalent if and only if one can pass from one to the other by a finite number of $K$-moves and their inverses.

To define the effect of a $K$-move on $[L, \bar{K}]$, we construct a diffeomorphism from $M_{L}$ to $M_{L^{\prime}}$. Let $\bar{K} \subset S_{3} \backslash L$ and let $\left(T_{i}\right)_{i \in\langle 1, n\rangle}$ be some disjoint toric tubular neighbourhoods of the $C_{i}$ s in $S_{3} \backslash \bar{K}$. KC $M_{L}$ clearly appears as $\bar{K}$ in $S_{3} \backslash\left(\cup_{i} T_{i}\right)$ because $M_{L}$ obtained from $S_{3} \backslash\left(\cup_{i} T_{i}\right)$ by gluing back the $T_{i}$ s via diffeomorphisms. Henceforth, our diffeomorphism can be defined on $N_{L}=S_{3} \backslash\left(\cup_{i} T_{i}\right)$ considered as a subset of $M_{L}$ and on the $T_{i}$ s by taking care on the way they are glued to $N_{L}$. This construction provides us with a natural definition of $K$-moves when $\bar{K} \neq \emptyset$ :

Definition 12. Let $L$ be a framed link in $S_{3}$ and $\bar{K}$ a framed $\phi^{3}$ diagram in $S_{3}$. Let $C$ be an unknotted component of framing $\varepsilon= \pm 1$. A K-move on $[L, \bar{K}]$ is defined as follows. We choose $D$ a disk in $S_{3}$ bounding $C$.

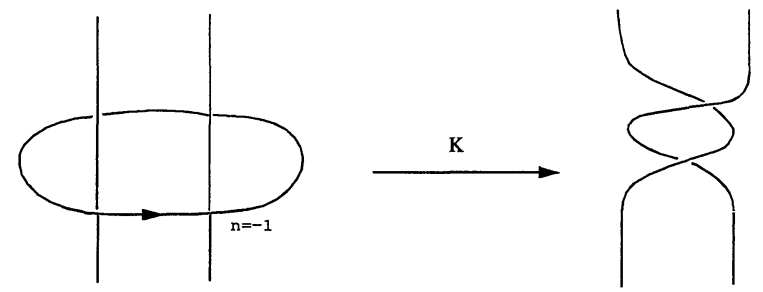

Fig. 3. Effect of a $K$-move: we remove the unknotted circle $(C)$ with framing 1 (in this example) 
- The effect of the K-move on L is the same as in Fenn and Rourke's definition (cf. Definition 11).

- The effect of a K-move on $\bar{K}$ is to twist all lines that pierce $D$ of $-2 \pi \varepsilon$ as if they belonged to $L$.

Let us remark that $\bar{K}^{\prime \prime}$ s framing is well defined in this procedure ${ }^{13}$.

Equivalence between $[L, \bar{K}]$ and $\left[L, \bar{K}^{\prime}\right]$. We shall now describe the set of graphs $\bar{K}^{\prime}$ in $S_{3} \backslash L$ such that $[L, \bar{K}] \simeq\left[L, \bar{K}^{\prime}\right]$. Surgery along $L$ in $\left[S_{3}, \bar{K}\right]$ (respectively $\left[S_{3}, \bar{K}^{\prime}\right]$ ) produces $M_{L}$ and $K$ (respectively $\left.K^{\prime}\right)$. $[L, \bar{K}]$ and $\left[L, \bar{K}^{\prime}\right]$ are equivalent if and only if $K$ is isotopic to $K^{\prime}$. Obviously, we mean that the isotopy is compatible with colourings of the graphs! If we consider $M_{L}$ as $N_{L}$ and the $T_{i}$ s glued via diffeomorphisms, we can decompose the isotopy in two phases:

- The isotopy takes place in $N_{L}$.

- The isotopy goes through the glued tori.

The first case is not a problem: it can be translated into an isotopy in $S_{3} \backslash \cup_{i} T_{i}$ ! Let us describe more precisely the second case.

We can suppose that during this phase, only one of the $T_{i}$ is concerned and nothing happens in $N_{L}$. Let $t_{1}$ and $t_{2}$ be the initial and final times of this isotopy. Consider $K(t)$ the position of the $\phi^{3}$ diagram at time $t$ and parts which move between $t_{1}$ and $t_{2}$. These can be located in a neighbourhood of the glued $T_{i}$ because we assumed only one torus is concerned. Vertices can be kept fixed during the move. Henceforth, we study the deformation of a single line of the graph. At $t \in] t_{1}, t_{2}[$, the intersection of this line with $T$ is an open curve $k(t)$. The reader is referred to Fig. 4. $k\left(t_{1}\right)$ can be continuously deformed into $k\left(t_{2}\right)$ by passing inside $T$ if and only if $k\left(t_{1}\right) k\left(t_{2}\right)^{-1}$ 's projection on $\partial T$ is null-homotopic in $T$. A loop drawn on a torus and homotopic to $\alpha(a)+\beta(b)$ with $(\alpha, \beta) \in \mathbf{Z}^{2}$ is trivial in $\pi_{1}(T)$ if and only if $\beta=0$. Translated into $N_{L}$, it means that the curve must be of the form: $m\left(n_{i}\left(a^{\prime}\right)\right.$ $+\left(b^{\prime}\right)$ ), where $m \in \mathbf{Z}$. This describes an elementary step in the equivalence between $\bar{K}$ and $\bar{K}^{\prime}$ : one of $\bar{K}$ 's lines (say $C_{j}$ ) is brought near $C_{i}$ in $S_{3} \backslash L$, and we replace a little segment of this line by one which follows a parallel curve to $C_{i}$. To describe the change of framings of the lines we modified, we consider a curve $\gamma$ parallel to $C_{j}$ and examine its changes into the various moves we performed as depicted on Fig. 5. This is the analog of the $\beta$-move introduced in Fenn and Rourke's paper and
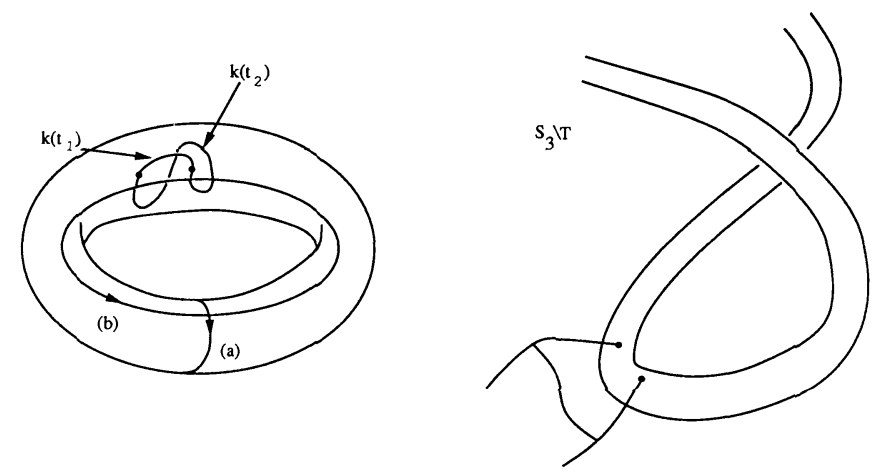

Fig. 4. If $k(t)$ denotes the position of the line we consider at time $t$ inside $T, k\left(t_{i}\right)$, and $k\left(t_{f}\right)$ are drawn on $\partial T$. The loop obtained by gluing these two paths has to be homotopically trivial in $T$

${ }^{13}$ Lines are considered as ribbons 


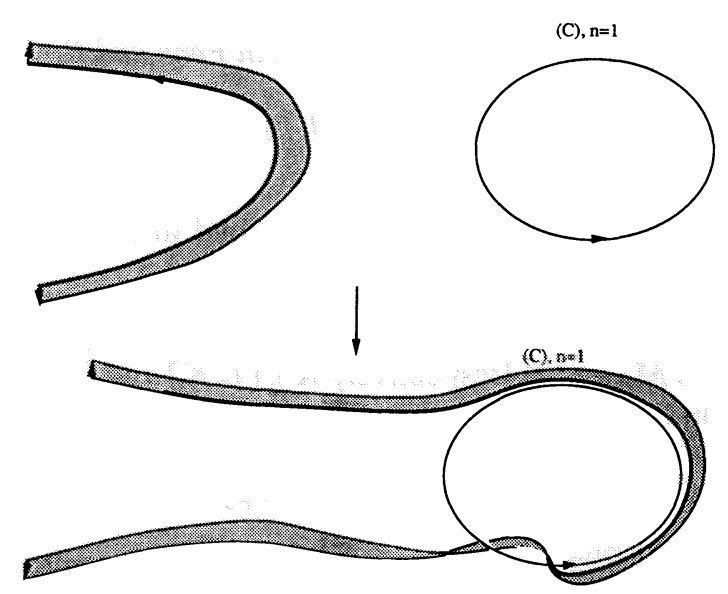

Fig. 5. Effect of a $\beta$-move on a framed line of $\bar{K}$. The line should be considered as a ribbon: it is dragged along a parallel curve to $C$

described originally by Kirby [26] as the $\mathcal{O}_{2}$ operation. The effect on the line as well as on its framing is the same. We shall denote it as a $\beta$-move performed on a line of $\bar{K}$. We have the following lemma:

Lemma 2. Two trivalent coloured framed graphs $\bar{K}$ and $\bar{K}^{\prime}$ of $S_{3} \backslash L$ are such that $[L, \bar{K}]$ is equivalent to $\left[L, \bar{K}^{\prime}\right]$ if and only if one can pass from $\bar{K}$ to $\bar{K}^{\prime}$ by a finite sequence of the following operations:

- Isotopies in $S_{3} \backslash L$.

- Performing a $\beta$-move on one line of $\bar{K}$ around one component of $L$ or its inverse.

Equivalence Criterion. This gives us the complete set of moves which we need to decide whether two surgery presentations $[L, \bar{K}]$ and $\left[L^{\prime}, \bar{K}^{\prime}\right]$ are equivalent in the sense of Definition 9. The theorem is:

Theorem 3. Two surgery presentations $[L, \bar{K}]$ and $\left[L^{\prime}, \bar{K}^{\prime}\right]$ are equivalent in the sense of Definition 9 if and only if one can relate them by a finite number of $K$-moves, isotopies in $S_{3}, \beta$-moves on a line of $\bar{K}$ around a component of the surgery link or their inverses.

To check that the partition function is invariant under these moves, we need the effect of these moves on the number $\sigma(L)$.

Effect of Kirby's Moves on $\sigma(L)$. As explained by Kirby, and also by Fenn and Rourke, adding an unknotted component $C$ of $L$, unlinked with any line of $L \backslash C$ (special $K$-move) induces the following changes on $W_{L}$ :

$$
\begin{gathered}
\varepsilon=1: W_{L} \mapsto W_{L}+P_{2}(\mathbf{C}), \\
\varepsilon=-1: W_{L} \mapsto W_{L}+\overline{P_{2}(\mathbf{C}),}
\end{gathered}
$$

where $\varepsilon= \pm 1$ denotes $C$ 's framing. We simply perform connected sum of $W_{L}$ with $P_{2}(\mathbf{C})$ or $\overline{P_{2}(\mathbf{C})}$ depending on $C$ 's framing. When we delete $C$ with framing $\varepsilon$, we get $\sigma\left(L^{\prime}\right)=\sigma(L)-\varepsilon$ ! On the other hand, performing a $\beta$-move along a component of $L$ does not change $W_{L}$ and it is obvious that performing such a $\beta$-move on a line belonging to $\bar{K}$ has no effect on $W_{L}$. This will be enough to study the behaviour of the partition function of our surgery presentation under various moves. 


\subsection{Topological Invariance of the Partition Function}

Let us check that the partition function associated with some surgery presentation does not depend on the representative of an equivalence class in the sense of Definition 9.

We shall examine the different cases successively starting from the isotopies in $S_{3}$, then studying the $K$-moves and finally looking at the case of $\beta$-moves. The influence of $\sigma(L)$ will be discussed at the end.

Isotopies in $S_{3}$. Let $\left[L^{\prime}, \bar{K}^{\prime}\right]$ be isotopic to $[L, \bar{K}]$ in $S_{3}$. As we notice in Appendix B, $Z\left[S_{3}, L_{\mathscr{C}}, \bar{K}\right]=Z\left[S_{3}, L_{\mathscr{C}}^{\prime}, \bar{K}^{\prime}\right]$. Clearly $\sigma(L)=\sigma\left(L^{\prime}\right)$, therefore:

$Z_{s}[L, \bar{K}]=Z_{s}\left[L^{\prime}, \dot{\bar{K}}^{\prime}\right]$.

Invariance Under K-Moves. Let us denote by $C_{\varepsilon}$ the unknotted component of $L$ with framing $\varepsilon= \pm 1$ we would like to delete from $L$. We compute the following expression:

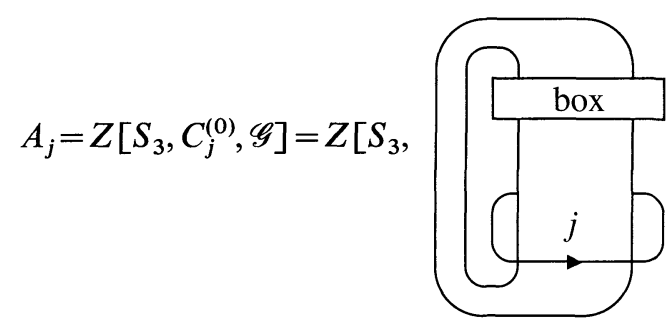

where $C_{j}^{(0)}$ has color $j$ and framing zero ${ }^{14}$ and $\mathscr{G}$ is a colored $\phi^{3}$ diagram which represents $L \backslash C$ and $\bar{K}$. An isotopy in $S_{3}$ has been used to carry $\left[C_{j}^{(0)}, \mathscr{G}\right]$ into the above form.

We assume that $m$ lines are running through a disk with boundary $C$. We have introduced a suitable basis of $H_{0,2 m}$ :

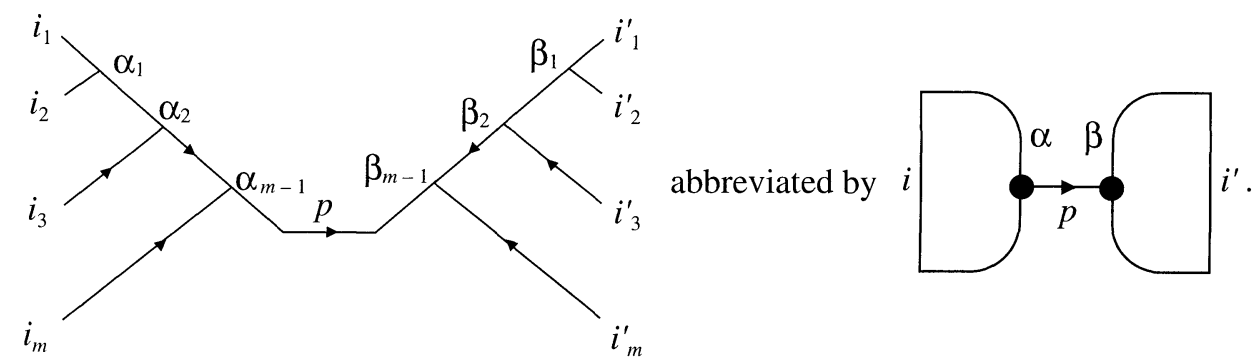

The following expression can be inferred from functoriality in $S_{3}$ along a two sphere $S_{2}$ :
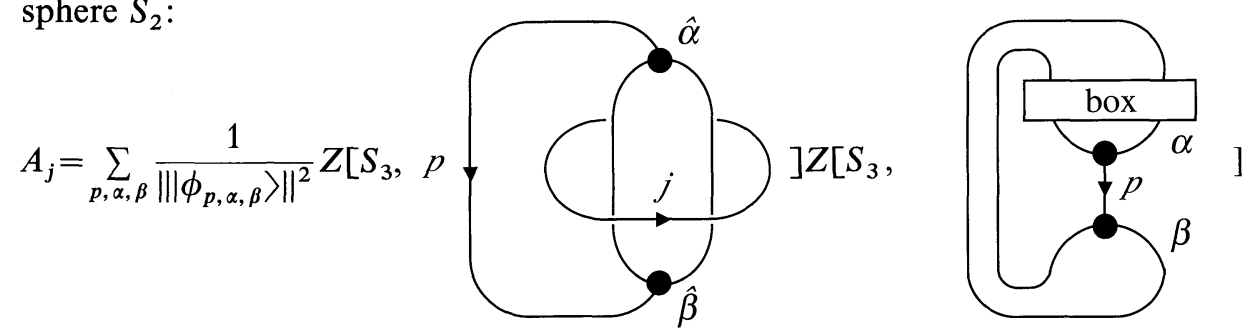

${ }^{14}$ With our choice of a projection, it coincides with normal framing ... 
By functoriality along $S_{2}$ in $S_{3}$, insertion of $C_{j}^{(0)}$ around a line colored by $p$ is equivalent to deleting line $C_{j}^{(0)}$ and multiplying the partition function by $S_{p}^{j} / S_{0}^{p}$ (see formula 43 for details). With topological invariance in $S_{3}$, it implies that:
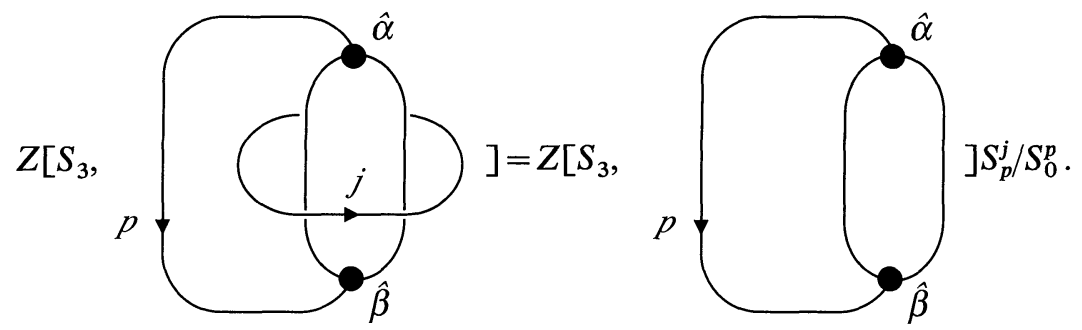

Let us go back to our surgery computation. The partition function of the surgery presentation $[L, \bar{K}]$ is a sum over all colorings of $L$. We split it into a sum over $C$ 's coloring and $L \backslash C$ 's one. Let us assume that we have fixed a coloring of $L \backslash C$ and we denote by $\mathscr{G}$ the union of $\bar{K}$ and $L \backslash C$ with their colorings. We are interested into:

$$
\begin{aligned}
\tilde{Z} & =\sum_{j} A_{j} \times\left(T^{\varepsilon} S\right)_{j}^{0} e^{2 \pi i \varepsilon c / 24} \\
& =\sum_{j, k} A_{j} S_{0}^{k} e^{-2 \pi i \varepsilon\left(h_{k}-c / 8\right)} S_{k}^{j} .
\end{aligned}
$$

We replace $A_{j}$ by the expression obtained above and find:

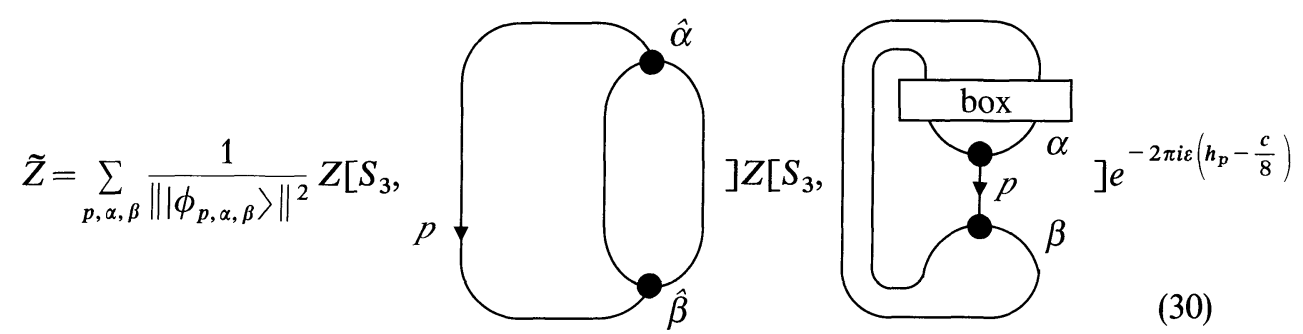

where we used that $S^{2}=C$.

Now we turn on the case of $L^{\prime}$. Using an isotopy in $S_{3}$, we see that the graph $\mathscr{G}$ introduced above changes and the new situation is described by:

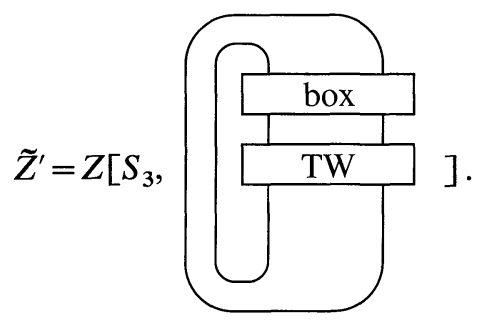

The TW box contains the twist mentioned above. We call the new graph $\mathscr{G}^{\prime}$. Here, framings are modified. To compute the partition function with a fixed coloring of $L^{\prime}$, we introduce a two-sphere with $2 m$ marked points to isolate the TW box from 
the rest. Henceforth, we only have to compute:

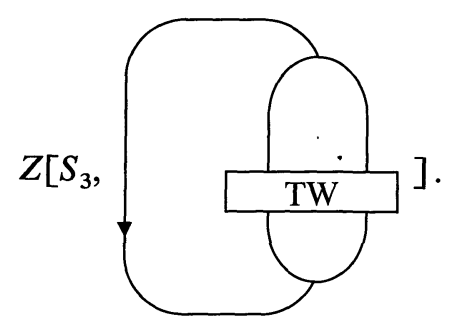

The framing of a line of $L^{\prime}$ is the same outside the TW box as it was in $L$ but inside this box, each line should be considered as a ribbon turning around the other lines as on Fig. 6. To compute $\bar{Z}^{\prime}$, we first go back to the normal framing and this introduces a phase

$$
\exp \left(-2 \pi i \varepsilon \sum_{l=1}^{q} h_{i_{l}}\right) \text {. }
$$

Then we compute the partition function by "de-twisting" near each vertex thanks to the identity:

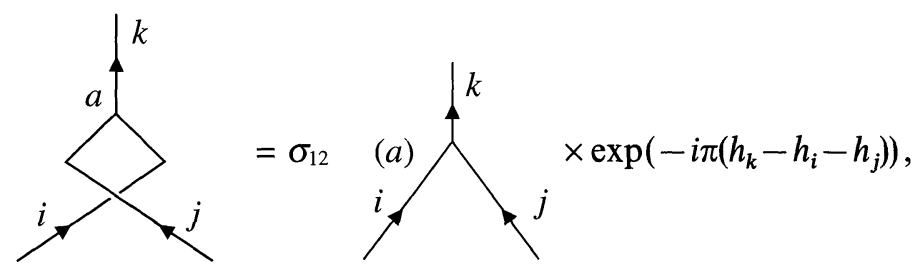

and the one obtained by changing the over-crossing into an under-crossing. Finally, we find after an obvious computation:

$$
\tilde{Z}^{\prime}=\tilde{Z} \times e^{-2 \pi i \varepsilon c / 8} .
$$

Comparing this with Eq. (30) shows that:

Lemma 3. Let $[L, \bar{K}]$ be a surgery presentation and $\left[L^{\prime}, \bar{K}^{\prime}\right]$ obtained from by a $K$-move on $[L, \bar{K}]$, then:

$$
Z_{s}[L, \bar{K}]=Z_{s}\left[L^{\prime}, \bar{K}^{\prime}\right] \times e^{2 i \pi\left(\varepsilon+\sigma\left(L^{\prime}\right)-\sigma(L)\right) c / 8} .
$$

We shall see in a while that the phase factor is indeed 1.

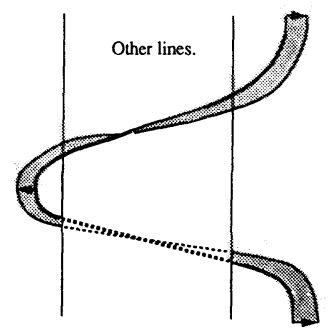

Fig. 6. Shape of a line inside the TW box. It is considered as a ribbon to define its framing 
Invariance Under $\beta$-Moves on $\bar{K}$. Let us denote by $\bar{K}^{\prime}$ the $\phi^{3}$ diagram obtained by performing a $\beta$-move on the line $j$ around component $i$ of $L$. We prove that $Z_{s}[L, \bar{K}]=Z_{s}\left[L, \bar{K}^{\prime}\right]$ using $K$-moves and isotopies. The proof is quite similar to the proof that $\beta$-moves on $L$ can be obtained from $K$-moves in Fenn and Rourke's paper [27]. More precisely, it can be straightforwardly adapted by considering that $K$-and $\beta$-moves act on $\bar{K}$ 's lines as we explained above. We then use Lemma 3 to see that a phase factor $\exp \left(2 \pi i c\left(\sigma\left(L^{\prime}\right)-\sigma(L)\right) / 8\right)$ arises $(\varepsilon= \pm 1$ contributions cancel). But in this case $\sigma(L)=\sigma\left(L^{\prime}\right)$ and therefore, we have:

Lemma 4. If $\bar{K}$ and $\bar{K}^{\prime}$ are related by a $\beta$-move performed along some component of $L$, then $Z_{\mathrm{s}}[L, \bar{K}]=Z_{\mathrm{s}}\left[L, \bar{K}^{\prime}\right]$.

Topological Invariance. To conclude, let us show that there is no phase factor in formula (32). Exactly as in the previous subsection, we can compute the phase appearing in a $\beta$-move performed along a component of $L$ : it appears to be zero because $\sigma(L)=\sigma\left(L^{\prime}\right)$. In the case of a special $K$-move, $\sigma\left(L^{\prime}\right)-\sigma(L)=-\varepsilon$ and therefore, the phase is one in this case. As special $K$-moves, $\mathcal{O}_{2}$ moves performed along lines of $\bar{K}$ and $L$ generate all $K$-moves, it proves that $Z_{s}[L, \bar{K}]$ is invariant under all Kirby moves:

Theorem 4. The partition function of a surgery presentation $[L, \bar{K}]$ only depends on the diffeomorphism class of $\left[M_{L}, K\right]$. It is a topological invariant for boundaryless manifolds decorated with a colored $\phi^{3}$ framed graph.

\section{Checking the Axioms of RTFTs}

In this section, we define the operator associated with manifolds with non-empty boundary and we check that this correspondence satisfies all axioms of a projective topological field theory.

\subsection{Definition of Operators}

Let us consider $M$ a three-manifold with boundary $\partial M$ interpolating between $N$ and $N^{\prime}$. We would like to associate with it an element of $\mathscr{L}\left(H_{N}, H_{N^{\prime}}\right)$. As we said before, for each graph $\mathscr{G}$, we have defined a basis of $H_{N}$ the elements of which are indexed with disjoint unions of decorated handlebodies. We shall define:

$$
\phi[M]\left|\phi_{i}\right\rangle=\sum_{f} \frac{Z\left[T_{i} \# M \# \widehat{T}_{f}\right]}{\left\langle\phi_{f} \mid \phi_{f}\right\rangle}\left|\phi_{f}\right\rangle,
$$

where $\phi_{i}\left(\phi_{f}\right.$ respectively) denotes $\left[T_{i}, \mathscr{G}_{i, \mathscr{C}_{i}}\right]\left(\left[T_{f}, \mathscr{G}_{f, \mathscr{C}_{f}}\right] \text { respectively }\right)^{15}$. In order this definition be consistent with Subsect. 2.1 we need to check that going from $\mathscr{G}$ to $\mathscr{G}^{\prime}$ introduces the right matrix, that is to say:

$$
\begin{aligned}
& Z\left[\left(T_{i}, \mathscr{G}_{i, \mathscr{C}_{i}}\right) \# M \#\left(T_{f}, \mathscr{G}_{f, \hat{\mathscr{C}}_{f}^{\prime}}^{\prime}\right)\right] \\
& =\sum_{\mathscr{C}_{f}} P\left(\mathscr{G}_{f}^{\prime}, \mathscr{G}_{f}\right)_{\mathscr{C}_{f}^{\prime}, \mathscr{C}_{f}} Z\left[\left(T_{i}, \mathscr{G}_{i, \mathscr{C}_{i}}\right) \# M \#\left(T_{f}, \mathscr{G}_{f, \hat{\mathscr{C}}_{f}}\right)\right], \\
& Z\left[\left(T_{i}, \mathscr{G}_{i, \mathscr{C}_{i}^{\prime}}^{\prime}\right) \# M\left(T_{f}, \mathscr{G}_{f, \hat{\mathscr{C}}_{f}}\right)\right] \\
& =\sum_{\mathscr{C}_{i}} P\left(\mathscr{G}_{i}^{\prime}, \mathscr{G}_{i}\right)_{\mathscr{C}_{i}, \mathscr{C}_{i}}^{*} Z\left[\left(T_{i}, \mathscr{G}_{i, \mathscr{C}_{i}}\right) \# M \#\left(T_{f}, \mathscr{G}_{f, \hat{\mathscr{C}}_{f}}\right)\right] .
\end{aligned}
$$

15 These are orthogonal bases 
It is enough to check these formulas for $F$ and $B$ moves since any change from $\mathscr{G}$ to $\mathscr{G}^{\prime}$ is obtained by a finite sequence of such elementary moves. Let us give the argument for $F$ moves, the other cases being treated in the same way. We can find in $T_{i}$ a three-ball $B_{3}$ such that its intersection with $\mathscr{G}_{i}^{\prime}$ and $\mathscr{G}_{i}$ looks as:
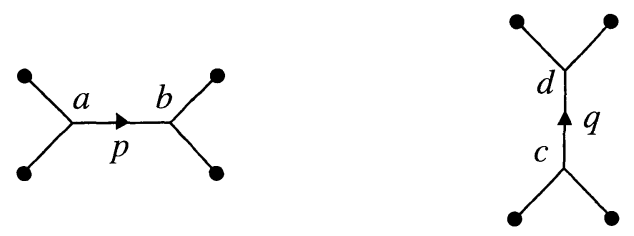

But functoriality along $S_{2}$ holds in any closed three-manifold (see Lemma 13) and therefore:

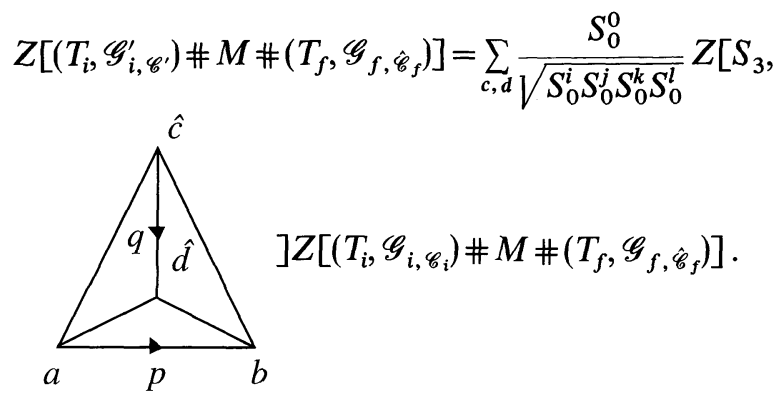

Explicitly evaluating the tetrahedron through the transfer matrix method gives us:

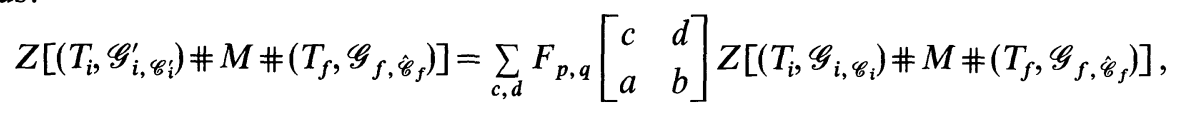

where $(q, c, d)$ are taken into account in $\mathscr{C}_{i}$ and $(p, a, b)$ into $\mathscr{C}_{i}^{\prime}$, the only difference between these two colorings being related to $(q, c, d)$ and $(p, a, b)$. Performing such a move on an outgoing state produces

$$
F_{\hat{p}, \hat{q}}\left[\begin{array}{ll}
\hat{c} & \hat{d} \\
\hat{a} & \hat{b}
\end{array}\right]=F_{p, q}\left[\begin{array}{ll}
c & d \\
a & b
\end{array}\right]^{*},
$$

and this proves our consistency formulas (33) and (34). We can then define:

Definition 13. Let $\left|\phi_{i}\right\rangle$ and $\left|\phi_{f}\right\rangle$ be two states belonging to $H_{N}$ and $H_{N}^{\prime}$ respectively which are associated with decorated handlebodies, then:

$$
\phi[M]\left|\phi_{i}\right\rangle=\sum_{f} \frac{Z\left[T_{i} \# M \# \hat{T}_{f}\right]}{\left\langle\phi_{f} \mid \phi_{f}\right\rangle}\left|\phi_{f}\right\rangle .
$$

Before checking all properties of this correspondence, let us remark that $\phi[M]$ is indeed a topological invariant object.

Let $M$ and $M^{\prime}$ be two isomorphic manifolds. From the definition of $\phi[M]$ and $\phi\left[M^{\prime}\right]$ 's matrix elements as functions of boundaryless glued manifolds, it is clear that $T_{i} \# M \# \widehat{T}_{f}$ and $T_{i} \# M^{\prime} \# \widehat{T}_{f}$ are diffeomorphic. Gluing of handlebodies are made via the parametrization of the boundary and the compatibility condition at the boundary appearing in Definition 2 precisely ensures the possibility of extending the diffeomorphism to the whole glued manifold! Therefore: 
Lemma 5. Let $M$ and $M^{\prime}$ be two isomorphic decorated three-manifolds, then $\phi[M]=\phi\left[M^{\prime}\right]$.

\subsection{Unitarity Axioms}

Let $M$ be a manifold interpolating between $N$ and $N^{\prime}$ and $\hat{M}$ with the reversed orientation, interpolating between $N^{\prime}$ and $N$. We compute $\left\langle\phi_{f}|\phi[M]| \phi_{i}\right\rangle$ $=\left[T_{i} \# M \# \hat{T}_{f}\right]$ and we have: $\left\langle\phi_{i}|\phi[\hat{M}]| \phi_{f}\right\rangle=Z\left[T_{f} \# \hat{M} \# \hat{T}_{i}\right]$. We know that:

$$
T_{i} \# \widehat{M \#} \widehat{T}_{f}=T_{f} \# \hat{M} \# \widehat{T}_{i},
$$

and therefore, we are led to computing $Z[\hat{X}]$ in terms of $Z[X]$, where $X$ is a decorated manifold without boundary. Such a manifold is obtained from the decorated sphere $\left[S_{3}, \bar{K}\right]$ by surgery along some particular link $L$. Let us start from $S_{3}$ and perform a surgery along the link $\widetilde{L}$ obtained from $L$ through an orientation reversing symmetry and with the opposite framing. Let $\hat{K}$ denote the graph obtained from $\bar{K}$ by an orientation reversing symmetry ${ }^{16}$ and turning its coloring into the opposite, then $[\widetilde{L}, \hat{K}]$ produces $\hat{X}$ : the orientation is reversed and the coloring of the decoration has turned into its opposite. Moreover, it is clear that $\sigma(\widetilde{L})=-\sigma(L)$. We have defined:

$$
Z_{s}[L, \bar{K}]=e^{-2 \pi i c \sigma(L) / 8} \sum_{\mathscr{C}}\left[\prod_{k=1}^{n}\left(\left(e^{2 \pi i c / 24} T\right)^{n_{k}} S\right)_{\mathscr{C}_{k}}^{0}\right] Z\left[S_{3}, L_{\mathscr{C}}, \bar{K}\right],
$$

and our orientation reversing operation produces:

$$
e^{2 \pi i c \sigma(L) / 8} \sum_{\mathscr{C}}\left[\prod_{k=1}^{n}\left(\left(e^{2 \pi i c / 24} T\right)^{-n_{k}} S\right)_{\mathscr{C}_{k}}^{0}\right] Z\left[S_{3}, \tilde{L}_{\mathscr{C}}, \hat{\bar{K}}\right] .
$$

But, from the definition of invariants in $S_{3}$ (see Appendix B, Sect. B.2.1):

$$
Z\left[S_{3}, \tilde{L}_{\hat{\mathscr{C}}}, \hat{\bar{K}}\right]=Z\left[S_{3}, L_{\mathscr{C}}, \hat{K}\right]^{*} .
$$

Moreover $\left(T^{p} S\right)_{j}^{0}=\left(\left(T^{-p} S\right)_{j}^{0}\right)^{*}$ and therefore, we find that:

$$
Z_{s}[L, \bar{K}]=\left(e^{2 \pi i c \sigma(L) / 8} \sum_{\mathscr{C}}\left[\prod_{k=1}^{n}\left(\left({ }^{2 \pi i c / 24} T\right)^{-n_{k}} S\right)_{\mathscr{C}_{k}}^{0}\right] Z\left[S_{3}, \tilde{L}_{\mathscr{C}}, \hat{K}\right]\right)^{*}
$$

which means that $Z[\hat{X}]=Z[X]^{*}$ ! Finally:

Lemma 6. $\phi[\hat{M}]=(\phi[M])^{\dagger}$.

\subsection{Projective Functoriality in any 3-Manifold}

Let $M_{1}$ and $M_{2}$ interpolating between $N$ and $N_{i}$ and $N_{i}$ and $N^{\prime}$ respectively, we would like to check projective functoriality. We check it on matrix elements which is equivalent to considering that $N=N^{\prime}=\emptyset$. Taking this point of view shows that the relevant objects are $\left|M_{1}\right\rangle=\phi\left[M_{1}\right] \cdot 1$ and $\left\langle M_{2}\right|=\phi\left[M_{2}\right]$. We thus have to prove the existence of $\mu\left(M_{1}, M_{2}\right) \in \mathbf{C}$ such that:

$$
Z\left[M_{1} \# M_{2}\right]=\mu\left(M_{1}, M_{2}\right) \sum_{\mathscr{C}} \frac{Z\left[\left(T_{g}, \mathscr{G}_{\mathscr{C}}\right) \# M_{2}\right] Z\left[M_{1} \#\left(\widehat{T}_{g}, \mathscr{G}_{\hat{\mathscr{C}}}\right)\right]}{\|\left|T_{g}, \mathscr{G}_{\mathscr{C}}\right\rangle \|^{2}} .
$$

16 As for $L \ldots$ 
The sum over $\mathscr{G}$ 's colorings is a sum over the elements of a basis of $H_{N_{i}}$. Each partition function that appears in this formula is to be computed using some appropriate surgery presentation. As we shall see in Subsect. 4.3.2, there exist some nice surgery presentations for $M_{1} \#\left[\widehat{T}_{g}, \mathscr{G}_{\mathscr{G}}\right]$ and $\left[T_{g}, \mathscr{G}_{\mathscr{G}}\right] \# M_{2}$ from which one can deduce a surgery presentation for $M_{1} \# M_{2}$. Then, we show that projective functoriality holds if and only if it holds in $S_{3}$ for special splittings, that is to say, a decomposition of the form $\left[S_{3}, K\right]=\left[T_{g}, K_{-}\right] \#\left[S_{3} \backslash T_{g}, K_{+}\right]$(see Subsect. 4.3.3).

4.3.1. Surgery and Boundaries. For the sake of simplicity, we shall only consider here the case $N_{i}$ connected. Other cases can be treated in the same way. The genus of $N_{i}=\Sigma$ is denoted by $g$ and we shall assume that $M_{1} \# M_{2}$ 's decoration intersects $\Sigma$ in $n$ points.

We shall use the following lemma:

Lemma 7. Any three-manifold with boundary $\Sigma$ can be obtained by surgery from $S_{3} \backslash T_{g}$.

Proof. This lemma can be inferred from the existence of Heegaard splittings for three manifolds with boundaries as described in [28]. The proof goes along the same lines as [25] and we leave it as an exercise for the reader.

We could as well have obtained a manifold with boundary $\Sigma$ by surgery from $T_{g}$ as explained by the following lemma:

Lemma 8. $S_{3} \backslash T_{g}$ is obtained by surgery from $T_{g}$ along the $\left(b_{j}\right)_{j \in\langle 1, g\rangle}$ with framing 0 . $T_{g}$ is obtained by surgery from $S_{3} \backslash T_{g}$ along the $\left(a_{j}\right)_{j \in\langle 1, g\rangle}$ with framing 0 .

Proof. The proof proceeds in two steps. First of all, we build an isomorphism from $S_{3} \backslash T_{1}$ onto the surgered handlebody. Then we make a recurrence on $g$ by gluing manifolds via a little disk. Both steps are elementary and left to the reader.

Application to RTFT need to distinguish between in and out-coming boundaries. This can be done quite easily as in this example: let $M \in$ hom $_{\mathbf{M a}}(\Sigma, \emptyset)$ be obtained from $S_{3} \backslash T_{g}$ by surgery along the framed link $L$. Then if $\left[\hat{T}_{g}, L^{\prime}\right]$ produces $\left[S_{3} \backslash T_{g}, L\right]$ by surgery along $C, M$ is obtained from $\hat{T}_{g}$ by surgery along $\left[L^{\prime}, C\right]$. Clearly $\hat{T}_{g} \in \operatorname{hom}_{\mathrm{Ma}}(\Sigma, \emptyset)$.

4.3.2. Clever Surgery Presentations. In our case, we $\left[M_{2}, K_{2}\right]$ is obtained from $\left[S_{3} \backslash T_{g}, \bar{K}_{2}\right]$ by surgery along $L_{2}$. Therefore, $\left[\left(T_{g}, \mathscr{G}_{\mathscr{C}}\right) \#\left(M_{2}, K_{2}\right)\right]$ arises from $\left[S_{3}, \mathscr{G}_{\mathscr{C}} \# \bar{K}_{2}\right]$ by surgery along $L_{2}$ now considered as a framed link in $S_{3}$.

Now, $\left[M_{1}, K_{1}\right]$ comes from $\left[T_{g}, \bar{K}_{1}\right]$ by surgery along $L_{1}$. Turning $\widehat{S_{3} \backslash T_{g}}$ into $T_{g}$ using surgery along $C \subset \widehat{S_{3} \backslash T_{g}}$ shows that $\left[\widehat{S_{3} \backslash T_{g}}, \bar{K}_{1}^{\prime}\right]$ gives us $\left[M_{1}, K_{1}\right]$ by surgery along $\left[L_{1}^{\prime}, C\right]$, where $L_{1}^{\prime}$ (respectively $\bar{K}_{1}^{\prime}$ ) denotes $L_{1}$ 's (respectively $K_{1}^{\prime}$ 's) antecedant in $\widehat{S_{3} \backslash T_{g}}$. Henceforth $\left[M_{1} \# \widehat{T}_{g}, K_{1} \# \mathscr{G}_{\hat{\varepsilon}}\right]$ is obtained from $\left[S_{3}, \bar{K}_{1}^{\prime} \# \mathscr{G}_{\hat{\mathscr{E}}}\right]$ by surgery along $\left[C, L_{1}^{\prime}\right]^{17}$. Finally, we end up with the following expressions:

$$
\begin{aligned}
Z\left[M_{1} \# \hat{T}_{g}, K_{1} \# \mathscr{G}_{\hat{\mathscr{C}}}\right]= & e^{-2 \pi i c \sigma_{1} / 8} \sum_{\mathscr{C}_{1}, \mathscr{C}} Z\left[S_{3}, \bar{K}_{1}^{\prime} \# \mathscr{G}_{\hat{\mathscr{C}}}, L_{1, \mathscr{C}_{1}}^{\prime}, C_{\mathscr{K}}\right] \\
& \times\left(\prod_{l=1}^{g} S_{\mathscr{K}_{l}}^{0}\right)\left(\prod_{j=1}^{m}\left(\left(e^{2 \pi i c / 24} T\right)^{m_{j}} S\right)_{\mathscr{C}_{1, j}}^{0}\right),
\end{aligned}
$$

$$
Z\left[T_{g} \# M_{2}, \mathscr{G}_{\mathscr{C}} \# K_{2}\right]=e^{-2 \pi i c \sigma_{2} / 8} \sum_{\mathscr{C}_{2}} Z\left[S_{3}, \mathscr{G}_{\mathscr{C}} \# \bar{K}_{2}, L_{2, \mathscr{C}_{2}}\right] \prod_{j=1}^{n}\left(\left(e^{2 \pi i c / 24} T\right)^{n_{j}} S\right)_{\mathscr{C}_{2, j}}^{0} .
$$

$17 \hat{S}_{3}$ can be confused with $S_{3}$ by taking care of its decoration: we have to change it by symmetry through a plane. Our notation is slightly abusive 


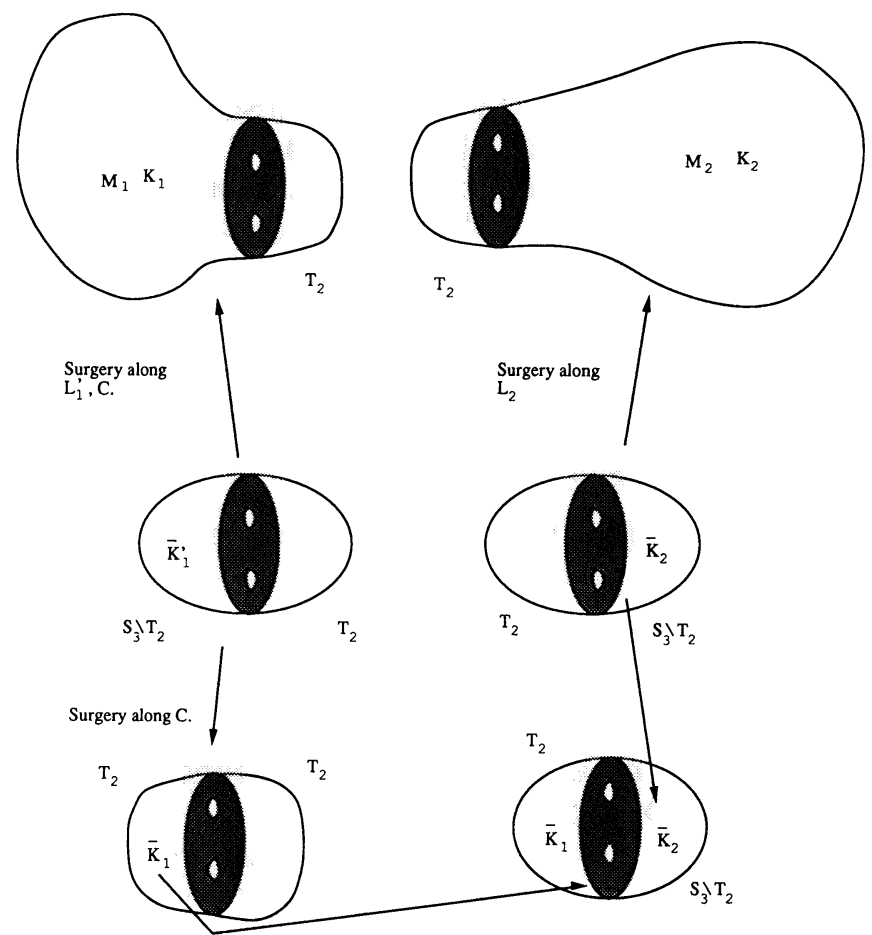

Fig. 7. Various surgery presentations used to prove projective functoriality. The surface along which we cut is shadowed

Figure 7 displays these various surgery presentations.

4.3.3. Proof of Functoriality. First of all, we sum over $C$ 's colorings $\mathscr{K}$ in formula (36). Thanks to the above remark, we get:

$$
Z\left[T_{g} \# \widehat{T}_{g}, \bar{K}_{1} \# \mathscr{G}_{\hat{\mathscr{C}}}, L_{1, \mathscr{C}_{1}}\right]=e^{-2 \pi i c \sigma_{g} / 8} \sum_{\mathscr{K}} Z\left[S_{3}, \bar{K}_{1}^{\prime} \# \mathscr{G}_{\mathscr{C}}, L_{1, \mathscr{C}_{1}}^{\prime}, C_{\mathscr{K}}\right] \prod_{l=1}^{g} S_{\mathscr{K}_{l}}^{0},
$$

where $\sigma_{g}$ is the phase factor appearing in Appendix B when one goes from $S_{3}$ to $T_{g} \# \widehat{T}_{g}$ by surgery along $C$.

Now, we can sum over $\mathscr{C}$ using formula (65) to recover a partition function in $S_{3}$

$$
Z\left[S_{3}, \bar{K}_{1} \# \bar{K}_{2}, L_{1}, L_{2}\right] \text {. }
$$

Putting all this together we find:

$$
\begin{aligned}
& e^{-2 \pi i c\left(\sigma_{1}+\sigma_{2}\right) / 8} \sum_{\mathscr{C}_{1}, \mathscr{C}_{2}} Z\left[S_{3}, \bar{K}_{1} \# \bar{K}_{2}, L_{1}, L_{2}\right] \\
& \quad \times\left(\prod_{k=1}^{n}\left(\left(e^{2 \pi i c / 24} T\right)^{n_{j}} S\right)_{\mathscr{C}_{2, k}}^{0}\right)\left(\prod_{j=1}^{m}\left(\left(e^{2 \pi i c / 24} T\right)^{m_{j}} S\right)_{\mathscr{C}_{1, j}}^{0}\right) .
\end{aligned}
$$

Fortunately, $\left[\left(L_{1}, L_{2}\right), \bar{K}_{1} \# \bar{K}_{2}\right]$ is nothing but a surgery presentation for $\left[M_{1}, K_{1}\right] \#\left[M_{2}, K_{2}\right]$ and finally we get:

$$
\left\langle M_{2} \mid M_{1}\right\rangle=Z\left[M_{1} \# M_{2}\right] e^{-2 \pi i c\left(\sigma_{1}+\sigma_{2}-\sigma_{1,2}\right) / 8},
$$

where $\sigma_{1,2}$ is the signature associated with the surgery presentation $\left[\left(L_{1}, L_{2}\right)\right.$, $\left.\bar{K}_{1} \# \bar{K}_{2}\right]$. Let us stress that partition functions on the left- and right-hand sides of 
this formula does not depend on the particular surgery presentations we choose to compute them. This provides us with an "explicit" expression for the cocycle:

$$
\mu\left(M_{1}, M_{2}\right)=e^{2 \pi i c\left(\sigma_{1}+\sigma_{2}-\sigma_{1,2}\right) / 8} .
$$

As we stressed before, this implies projective functoriality of our correspondence between interpolating 3-manifolds and operators acting on the $H_{N}$ s. To prove it precisely, it is convenient to go back to our definition of operators associated with manifolds with boundaries.

We consider $M_{1}$ and $M_{2}$ interpolating between $N$ and $N_{i}$ and $N_{i}$ and $N^{\prime}$ respectively, then let $T, T^{\prime}$, and $T_{i}$ be decorated handlebodies used to cap $M_{1,2}$ 's boundaries, we have ${ }^{18}$ :

$$
\begin{aligned}
Z & {\left[T \# M_{1} \# M_{2} \# \hat{T}^{\prime}\right] } \\
& =\mu\left(T \# M_{1}, M_{2} \# \hat{T}^{\prime}\right) \sum_{\mathscr{I}} \frac{Z\left[T \# M_{1} \# \hat{T}_{i, \hat{\mathscr{I}}}\right] Z\left[T_{i, \mathscr{I}} \# M_{2} \# \hat{T}^{\prime}\right]}{\left.\| T_{i}, \mathscr{I}\right\rangle \|^{2}}
\end{aligned}
$$

the number $\mu\left(T \# M_{1}, M_{2} \# \hat{T}^{\prime}\right)$ apparently depends on $T$ and $T^{\prime \prime}$ s decorations. However, to compute it, we use signatures of manifolds bounding $T \# M_{1} \# M_{2} \# T^{\prime}, T \# M_{1} \# \widehat{T}_{i}$, and $T_{i} \# M_{2} \# T^{\prime}$ regardless to their decorations. Henceforth it does not depend on $T, T^{\prime \prime}$ 's decorations. This enables us to consider Eq. (42) as a down to earth expression of the following lemma ${ }^{19}$

Lemma 9. Let $M_{1}$ and $M_{2}$ interpolate between $N$ and $N_{i}$ and $N_{i}$ and $N^{\prime}$ respectively, then there exists $\mu\left(M_{1}, M_{2}\right) \in \mathbf{C}$ such that:

$$
\phi\left[M_{1} \# M_{2}\right]=\mu\left(M_{1}, M_{2}\right) \times \phi\left[M_{2}\right] \circ \phi\left[M_{1}\right] .
$$

Cocycle condition on $\mu$ is clearly satisfied. Let us now explore some consequences of this important property.

\subsection{Consequences of Functoriality}

We shall examine two consequences of Lemma 9. Namely, we explain how to compute partition functions using Morse functions, topology changing amplitudes and a projective representation of modular groups $\operatorname{Diff}_{+}(N) / \operatorname{Diff}_{+, 0}(N)$ in $H_{N}$. We also discuss the relation with the one that arise in Moore and Seiberg's work and the relation to the phase appearing in Sect. 3. Let us warn the reader that we do not have clarified this point completely and we shall therefore be quite sketchy in this section. We only intend to describe the main problems. Finally, we prove in Sect. 4.4.3 that the gluing axiom is satisfied.

4.4.1. Morse Theory Computations. Let us consider $M$ a boundaryless decorated 3-manifold, we choose a Morse function $f$ which separates not only $M$ 's singular points but also singularities of its decoration. We decompose $M$ into slices $M_{k}$ with $k \in\langle 1, N\rangle . \quad M_{k}=f^{-1}\left(\left[t_{k}, t_{k+1}\right]\right)$ where $t_{k}$ is a regular value of $f$ and $M=M_{1} \# M_{2} \# \ldots \# M_{N}$. Thanks to Lemma 9, we can compute $M$ 's partition

18 In this formula $T_{i, \mathscr{I}}$ means that $T_{i}$ 's decoration is colored by $\mathscr{I}$

19 With $\mu\left(M_{1}, M_{2}\right)=\mu\left(T \# M_{1}, M_{2} \# \hat{T}^{\prime}\right)$ ! 
function as $Z[M]=\phi\left[M_{N}\right] \circ \ldots \circ \phi\left[M_{1}\right]$. Indeed, it is possible to choose $f$ in such a way that one has some well-defined slices near $f$ 's critical values. Moreover, if between two regular values of $f$, there is no singular value of $f$, then initial and final sections of these slices only differ by a diffeomorphism. We are thus led to computing the action of diffeomorphisms and topology changing amplitudes.

Let us focus on diffeomorphisms. Any PTFT provides a projective representation of the modular group

$$
\mathscr{M}(N)=\frac{\operatorname{Diff}_{+}(N)}{\operatorname{Diff}_{+, 0}(N)}
$$

on the Hilbert space $H_{N}$. Here, we have constructed a PRTFT based on a geometric category the objects of which are orientable closed surfaces with some marked points and a non-zero tangent vector at each point. A PTFT based on this category defines a projective representation of $M^{*}(g, n)$ (the modular group of a genus $g$ surface with $n$ punctures and a coordinate at each puncture) on $H_{g, n}$.

This group is generated by all non-trivial Dehn twists. For example, Dehn twists around one of the punctures, around non-trivial cycles (which generate the usual modular group). The interested reader can find a description of a complete set of generators of $M^{*}(g, n)$ in the work of Birman [29]. The idea is to define the action of the $S$ modular transformation on $H_{1,1}$ and the action of Artin's braid group on $H_{0,4}$. Thanks to Birman's work, this defines the action of $M^{*}(g, n)$ on $H_{g, n}$ as soon as we know the cocycle.

In a topological field theory, any element $f$ of the modular group can be -represented by a particular manifold $M(f)$ the gluing of which defines the action of $f$. This manifold is defined as follows: if we want to define the action of $f \in \operatorname{Diff}_{+}(N) / \operatorname{Diff}_{+, 0}(N)$ on $H_{N}$, then $M(f)$ has to interpolate between $N$ and $N$. In order to define $M(f)$, one can consider a cylinder $N \times[0,1]$ with parametrization $\varphi$ at one end and $f \circ \varphi$ at the other end, such that $M_{1} \# M(f) \# M_{2}$ is isomorphic to $M_{1} \#_{f} M_{2}$ for any manifold $M_{1,2}$ with $N \subset \partial M_{1,2}$. To illustrate this point, we compute the action of $S$ on $H_{1,1}$, the action of a Dehn twist around one puncture and the action of half a Dehn twist around two punctures in the case of the fourpunctured sphere.

- Let us take $s$ the modular transformation of the torus which sends $(a)$ on $(b)$ and (b) on $-(a)$. We denote $\phi[M(s)]=S$. Let $\left(\left|\chi_{i, a}^{j}\right\rangle\right)_{i, j, a}$ be the usual basis of $H_{1,1}$, we have:

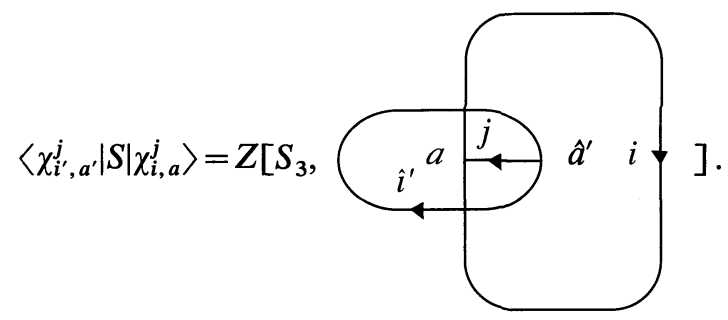

Thanks to Moore and Seiberg's equations, we find:

$$
\left\langle\chi_{i^{\prime}, a^{\prime}}^{j}|S| \chi_{i, a}^{j}\right\rangle=\sqrt{\frac{S_{0}^{j}}{S_{0}^{0}}} S(j)_{(i, a)}^{\left(i^{\prime}, a^{\prime}\right)},
$$

where $S(j)$ is the modular transformation matrix. 

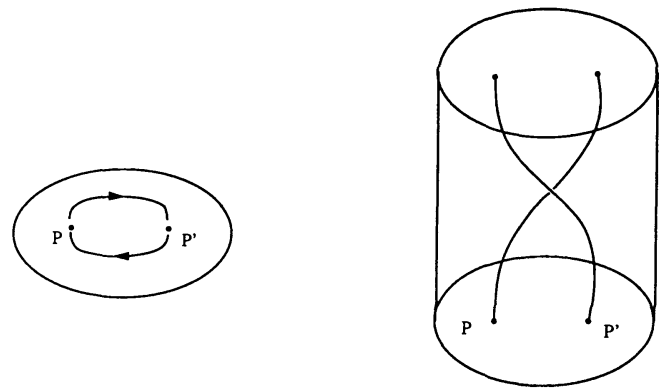

Fig. 8. Representation of half a Dehn twist on two points $P$ and $P^{\prime}$ located in a disk $D$ by a cylinder in which lines associated with these points are braided

- The action of a Dehn twist of $2 \pi n$ performed along a simple curve around $P$ but trivial in $\Sigma-\{P\}$ is represented by a cylinder $\Sigma \times[0,1]$ where the line passing by $P$ has a framing twisted by $n$ units. Therefore, any vector where the line passing by $P$ carries an index $i$ is multiplied by $\exp \left(2 \pi i n h_{i}\right)$. This is exactly the action of a Dehn twist around a point as it appears in Moore and Seiberg's paper.

- Next, let us consider two points $P, P^{\prime}$ in a disk $D_{2} \subset \Sigma$. We perform half a Dehn twist on these two points as indicated in Fig. 8. This diffeomorphism of the surface is associated with the cylinder $H$ in which the lines passing by $P$ and $P^{\prime}$ are braided as indicated in Fig. 8. On the four punctured sphere, we consider $|\phi(p, a, b)\rangle$ defined by:

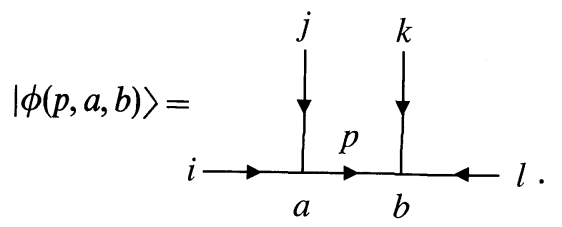

Of course, the braiding can be performed in two different ways giving rise to $H_{ \pm}$. Then after a short computation:

$$
\left\langle\phi\left(p^{\prime}, c, d\right)\left|\phi\left[H_{ \pm}\right]\right| \phi(p, a, b)\right\rangle=\frac{\sqrt{\prod_{k=1, \ldots, 4} S_{0}^{i_{k}}}}{S_{0}^{0}} B_{p, p^{\prime}}( \pm)\left[\begin{array}{ll}
c & d \\
a & b
\end{array}\right] .
$$

It coincides with Moore and Seiberg's expressions.

It remains to check that in the case of a general surface and with any element of the modular group, the same phenomenon occurs. Indeed, Moore and Seiberg have defined a projective representation of the modular group $M^{*}(g, n)$ on $H_{g, n}$ using the $F, B, S$, and $T$ matrices. Let us suggest why the representation arising from our PRTFT and Moore and Seiberg's work coincide.

We shall prove coincidence for a generating set of $M^{*}(g, n)$. We shall not describe here all technical details, the computations being mostly quite simple and similar to the ones carried out in $[23,8]$. Cocycle problems will be analysed in the next section.

The case of Dehn twists around one puncture is clear. We only need to look at the modular group $M(g, n)$ of a surface with $n$ punctures but no coordinates at the puncture. We have the following exact sequence:

$$
1 \rightarrow \mathbf{Z}^{n} \rightarrow M^{*}(g, n) \rightarrow M(g, n) \rightarrow 1 .
$$


A set of generators of $M(g, n)$ is given by monodromies of the points on the surface and a set of generators of the ordinary modular group. The monodromies are closely related to the braid group on the genus $g$ surface. The following exact sequences hold:

- For $g \geqq 2$ :

$$
1 \longrightarrow C B_{n}(g) \longrightarrow M(g, n) \longrightarrow M_{g} \longrightarrow 1 \text {. }
$$

- For $g=1, n>1$ or $g=0, n>2$ :

$$
1 \longrightarrow \frac{C B_{n}(g)}{\text { center }} \longrightarrow M(g, n) \longrightarrow M_{g} \longrightarrow 1,
$$

where $C B_{n}(g)$ is the set of colored $n$-braids on a closed orientable surface of genus $g$ defined as the fundamental group of the configuration space:

$$
\left\{\left(z_{1}, \ldots, z_{n}\right) \in\left(\Sigma^{\prime \prime}\right)^{n} / \forall i \neq j, z_{i} \neq z_{j}\right\} \text {. }
$$

Moreover, if we denote Artin's braid group by $B_{n}$ and $B_{n}(g)$ the uncolored braid group of the genus $g$ surface, we have:

$$
1 \longrightarrow B_{n} \longrightarrow B_{n}(g) \longrightarrow\left(\pi_{1}\left(\Sigma^{\prime \prime}\right)\right)^{n} \longrightarrow 1
$$

where the kernel of each homomorphism is the normal closure of the image of the previous homomorphism.

To describe the arrow from $C B_{n}(g)$ to $M(g, n)$, let us consider $\gamma \in C B_{n}(g)$ and associate with it $f_{\gamma} \in M(g, n)$ defined as in [29]: if $\gamma(t)$ is the position of our $n$ points at time $t^{20}$, we can define a path $t \mapsto F_{t}$ in $\operatorname{Diff}_{+}\left(\Sigma_{g}^{\prime \prime}\right)$ such that $F_{t}$ sends $\gamma(0)$ on $\gamma(t)$ ( $\Sigma_{g}^{\prime \prime}$ is a genus $g$ surface with no punctures). $f_{\gamma}=F_{1}$ is considered as a diffeomorphism of $\Sigma^{\prime \prime}$ with $n$ marked points. Obviously, it is trivial in $M_{g}$. The manifold $M\left(f_{\gamma}\right)$ is easily constructed: it is a cylinder $\Sigma_{g}^{\prime \prime} \times[0,1]$ with the image of $\gamma$ in it. Computing $\phi\left[M\left(f_{\gamma}\right)\right]$ is quite simple: we only need to compute it for special braids: namely, the liftings of generators of Artin's braid group in $B_{n}(g)$, and braids obtained by driving one point along a generator of $\pi_{1}\left(\Sigma^{\prime \prime}\right)$. The first case has already been analyzed on the four punctured sphere. Thanks to projective functoriality, the result is obtained on any surface. Let us now turn to the second case; the fundamental group $\pi_{1}\left(\Sigma^{\prime \prime}\right)$ is generated by the $\left(a_{i}\right)$ and $\left(b_{i}\right)$ cycles. We only have to drive one of the points around a non-trivial cycle of $\Sigma^{\prime \prime}$. More complicated cases can be obtained by composing such elementary moves and using Artin's braid group. Thanks to projective functoriality, we are led to considering the two punctured torus. We use projective functoriality to cut our manifold into pieces. This gives the result for the particular elements of $M^{*}(1,2)$ we consider. We are thus able to find expressions for any representative of a lifting of $\left(\pi_{1}\left(\Sigma^{\prime \prime}\right)\right)^{n}$ into $M(g, n)$ and of an embedding of $C B_{n}$ into $M(g, n)$ both via $C B_{n}(g)$. The expressions we find are identical to the ones appearing in Moore and Seiberg's analysis up to powers of $\exp (2 \pi i c / 24)$.

Finally, let us study the action of the modular group $M_{g}$. This group is generated by Dehn twists around non-trivial loops of $\Sigma^{\prime \prime}$. More precisely, $M_{g}$ is generated by Dehn twists around the $\left(a_{i}\right),\left(c_{j}\right)$, and $\left(b_{i}\right)$ cycles. We leave it to the reader to compute matrix elements of operators representing these diffeomorphisms. Using projective functoriality, one easily sees that the case of $\left(a_{i}\right)$ and $\left(b_{i}\right)$ cycles reduces to the similar case on the torus where the result is easily shown to be true by direct computation in the case of the $(a)$ cycle, and by using the $s$ modular

${ }^{20} \mathrm{~A}$ braid is nothing but an isotopy class of paths in a configuration space 
transformation in the case of the $(b)$ cycle. A direct computation in the case of $\left(c_{j}\right)$ cycles gives the result.

Finally, both projective representations coincide on a generating set of $M^{*}(g, n)$ up to powers of $\exp (2 \pi i c / 24)$. The cocycle in both cases is also a power of this number. Let us now turn to cocycle problems.

4.4.2. About Projectivity.... Associated with projective representations arising from our PTFT and Moore and Seiberg's work is a series of cocycles belonging to $\mathrm{H}_{2}\left(M^{*}(g, n), \mathrm{Z}\right)$. First of all, we can wonder whether these cocycles coincide or not. Another question would be to find a nice interpretation of them. Answering this second question in a sufficiently powerful way should provide an answer to the first one.

As we indicated before, a PTFT is nothing but a true TFT defined on a central extension if Ma by an abelian group ${ }^{21}$. It would be nice to find a geometric interpretation of this integer valued degree of freedom, that is to say, to build a new geometric category $\mathbf{M a}^{\prime}=\mathbf{M a} \mathbf{a}_{e}$ on which the TFT could be defined naturally. Witten originaly argued $[3,30]$ that the corresponding degree of freedom is the framing of the three-manifold, that is to say the isotopy class of trivialization of $T M \oplus T M$, where $T M$ is $M$ 's tangent bundle. This idea has been explored by Atiyah [31]. It is however not so easy to define the framing of a three manifold with boundary. Nevertheless Atiyah has defined a cocycle for all modular groups $M_{g}$ and has shown the existence of a universal cocycle. We refer the reader to his paper for details. Such a notion also appears in Harer's paper [32, Sect. 5]. Let us state a natural conjecture.

Let $(f, h)$ be two diffeomorphisms of a genus $g$ surface without punctures, we define $\mu(f, h)$ to be $\mu(M(f), M(h))$ : it clearly defines a 2-cocycle on the modular group $M_{g}$. We conjecture that:

Conjecture. If $\Delta$ denotes the generator of $\mathrm{H}_{2}\left(\mathrm{M}_{g}, \mathrm{Z}\right)$ defined by Harer, then it coincides with Atiyah's universal cocycle and moreover:

$$
\mu(f, g)=\exp \left(2 \pi i \frac{c}{8} \Delta(f, g)\right) \text {. }
$$

However I do not know any precise investigation of these matters in the framework of RTFT. Clearly, a serious and explicit look at central extensions of the tower of Teichmüller modular groups is needed. I hope to clarify these problems sooner or later. This is clearly related to the phase we have introduced in Sect. 3: this arbitrary although natural phase fixing is at the origin of the projectivity of our TFT and fixes the form of the cocycle for modular groups.

4.4.3. Gluing Axiom. The gluing axiom is a consequence of functoriality and topological invariance:

Proposition. Given two categories Ma and $\mathbf{S p}$ as in Subsect. 1.1 and a functor $\boldsymbol{\Phi}$ between these categories such that:

$$
\begin{aligned}
H_{\hat{N}} & =\left(H_{N}\right)^{*}, \quad \phi[\hat{M}]=(\phi[M])^{\dagger}, \\
H_{N \cup N^{\prime}} & =H_{N} \otimes H_{N^{\prime}}, \quad \phi\left[M \cup M^{\prime}\right]=\phi[M] \otimes \phi\left[M^{\prime}\right], \\
H_{\phi} & =\mathbf{C},
\end{aligned}
$$

and $H_{N}$ only depends on the topology of $N$ and $\mathbf{\Phi}$ is topologically invariant, then it defines a TFT. 
Proof. We consider $M$ a manifold interpolating between $N_{1}$ and $N_{2}$. We suppose that two connected components of $N_{1}\left(N_{2}\right.$ respectively) are isomorphic to $N: N$ $C N_{1}$ and $N \subset N_{2}$. We can glue together these components building $M_{[N]}$. As usual, we directly check the gluing axiom on matrix elements. Therefore, we assume $M$ to interpolate between $N$ and $N . M_{[N]}$ will be denoted by $\tilde{M}$. We want to show that $Z[\tilde{M}]=\operatorname{Tr}(\phi[M])$. One can choose a Morse function on $\tilde{M}$ as indicated on Fig. 9. Using this presentation is equivalent to considering a manifold $\bar{M}$ interpolating between $N \cap N$ and $\emptyset$. But $\phi[M]$ and $\phi[\bar{M}]$ 's matrix elements are related by:

$$
\left\langle T_{g}, \mathscr{G}_{\mathscr{C}^{\prime}}|\phi[M]| T_{g}, \mathscr{C}_{\mathscr{C}}\right\rangle=\phi[\bar{M}]\left(\left|T_{g}, \mathscr{G}_{\mathscr{C}}\right\rangle \otimes\left|T_{g}, \mathscr{G}_{\mathscr{G}^{\prime}}\right\rangle\right) .
$$

Henceforth:

$$
\begin{aligned}
\operatorname{Tr}(\phi[M]) & =\sum_{\mathscr{C}} \frac{\left\langle T_{g}, \mathscr{G}_{\mathscr{C}}|\phi[M]| T_{g}, \mathscr{G}_{\mathscr{C}}\right\rangle}{\left\langle T_{g}, \mathscr{G}_{\mathscr{C}} \mid T_{g}, \mathscr{G}_{\mathscr{C}}\right\rangle} \\
& =\sum_{\mathscr{C}} \frac{\phi[\bar{M}]\left(\left|T_{g}, \mathscr{G}_{\mathscr{C}}\right\rangle \otimes\left|T_{g}, \mathscr{G}_{\mathscr{G}}\right\rangle\right)}{\left\langle T_{g}, \mathscr{G}_{\mathscr{C}} \mid T_{g}, \mathscr{G}_{\mathscr{C}}\right\rangle} .
\end{aligned}
$$

$\tilde{M}$ is obtained from $\bar{M}$ by gluing to it a cylinder $N \times[0,1]$ as indicated on Fig. 9 . We denote by $P$ the manifold we glue. It interpolates between $\emptyset$ and $N \cup N$. Topological invariance and $\phi[N \times[0,1]]=\mathbf{1}_{\boldsymbol{H}_{N}}$ implies:

$$
\left(\left\langle\mathscr{G}_{\mathscr{C}}\right| \otimes\left\langle\mathscr{G}_{\mathscr{G}}\right|\right) \phi[P] \cdot 1=\left\langle\mathscr{G}_{\mathscr{C}} \mid \mathscr{G}_{\mathscr{G}^{\prime}}\right\rangle \text {. }
$$

Finally, thanks to the orthogonality of our basis,

$$
\operatorname{Tr}(\phi[M])=\sum_{\mathscr{C}} \sum_{\mathscr{C}^{\prime}} \frac{\phi[\bar{M}]\left(\left|T_{g}, \mathscr{G}_{\mathscr{C}}\right\rangle \otimes\left|T_{g}, \mathscr{G}_{\mathscr{\mathscr { C }}}\right\rangle\right)}{\left(\left\langle T_{g}, \mathscr{G}_{\mathscr{G}} \mid T_{g}, \mathscr{G}_{\mathscr{C}}\right\rangle\right)^{2}}\left\langle T_{g}, \mathscr{G}_{\mathscr{C}} \mid T_{g}, \mathscr{G}_{\mathscr{\mathscr { C }}^{\prime}}\right\rangle .
$$

But this expression is nothing but $\phi[\bar{M}] \circ \phi[P] \cdot 1$ which is $Z[P \# \bar{M}]=Z[\tilde{M}]$. Henceforth: $Z[\tilde{M}]=\operatorname{Tr}(\phi[M])$.

In our context, we have shown that all axioms of a PRTFT were satisfied. Thus, one builds an extension $\mathbf{M a}_{e}$ and a functor from $\mathbf{M a} \mathbf{a}_{e}$ to $\mathbf{S p}$ which verifies the axioms of a TFT except the gluing axiom. Proposition 4.4.3 shows that indeed it does and therefore this proves that in a projective TFT we have the following property:

$$
\phi\left[M_{[N]}\right] \propto \operatorname{Tr}_{H_{N}}(\phi[M]) .
$$

\section{Conclusion}

To conclude, Witten's conjecture about the interpretation of Moore and Seiberg's equations as the equations of motion of RTFT is verified. Nevertheless, this does

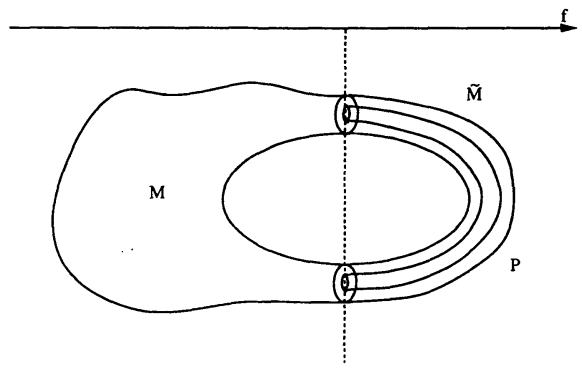

Fig. 9. A particular choice of a Morse function to compute $Z\left[M_{[N]}\right]$ 
not seem to illuminate the classification of RCFTs. Several questions remain unsolved:

- Various results about representations of modular groups remain to be collected in a single place and the relationship of all that stuff with TFT remains to be definitively clarified. This should give a clear interpretation of the phase $\exp (2 \pi i c / 24)$.

- Given a solution to Moore and Seiberg's equations, find a RCFT the conformal blocks of which define the same representations of all modular groups $M^{*}(g, n)$. Let us remark that the solution, when it exists, is far from being unique! Tensoring by any holomorphic CFT at $c=24$ leaves all monodromies invariant and therefore does not change the representation of the modular group. The unicity problem is thus connected to the classification of all holomorphic CFTs at $c \equiv 0(\bmod 24)$. The existence problem involves a kind of Riemann-Hilbert problem ${ }^{22}$ constrained by certain positivity and integrality requirements ${ }^{23}$. This problem is deeply rooted in number theory and remains unsolved. Finally, one has to reconstruct a chiral algebra, the representation theory of which will provide us with conformal blocks!

- Classify all solutions to Moore and Seiberg's equations. It has appeared that the representation theory of Quasitriangular Hopf algebras (among which are quantum groups) automatically built solutions to these equations. Moreover, Moore and Seiberg, using a reconstruction theorem of Deligne [34, 14] have proved that in a certain limit, solutions to Moore and Seiberg's equations were classified by representation theories of compact groups. However, such a program has not been completed in the general case mainly because any powerful reconstruction theorem à la Tannaka-Krein-Deligne is still lacking.

- A recent work by Baulieu and his collaborators [35] exhibits very exciting and mysterious relations between TFTs in $d, d+1$, and $d+2$ dimensions. Starting from 3D Chern-Simons theory and quantizing it by stochastic quantization naturally leads to considering Witten's topological field theory in 4 dimensions $[22,36]$. A similar connection also exists between 2D quantum gravity and 4D topological gravity. It would be extremely interesting to clarify this from an algebraic point of view. This would connect $3 \mathrm{D}$ topology to $4 \mathrm{D}$ geometry in a rather amazing way.

Unfortunately, none of these questions is solved at present time.

Acknowledgements. During this work, I benefited from many useful conversations with B. Julia. Various steps and previous versions of this work were presented to D. Arnaudon, M. Bauer, M. Picco and Ph. Roche. They gave me much useful advice. I also thank V. Jones for his interest and discussions. In June 1990, I had useful conversations with N. Reshetikhin on the issue of tetrahedral symmetry for $F$ matrices. Finally V.G. Turaev and N. Reshetikhin communicated to me useful references and a preliminary version of their work.

Note added. While correcting the proofs of our paper, we had an interesting discussion with D. Altschüler and A. Coste about their recent work [37] on topological invariants that arise from quasi-quantum groups related to orbifolds of holomorphic CFTs. As can be expected from our work, since $c \equiv 0(\bmod 8)$ in these CFTs, representations of modular groups that arise from their construction are not projective as they have checked in particular cases. It is an interesting exercise to compare their results for partition functions of lens spaces with ours in some simple cases. Both approaches coincide as one would expect.

${ }^{22}$ Given monodromies, find functions which precisely have these monodromies

${ }^{23}$ The reader will remember these problems in the context of the quest for modular invariant partition functions [33] 


\section{A. Technicalities around $\boldsymbol{F}$ Matrices}

The purpose of this appendix is to prove some technical properties of the $F$ matrices such as their tetrahedral symmetry and their unitarity.

\section{A.1. Tetrahedral Symmetry of the F Matrix}

Let us define:

$$
W_{p, q}\left[\begin{array}{ll}
c & d \\
a & b
\end{array}\right]=\frac{\sqrt{S_{0}^{i} S_{0}^{j} S_{0}^{k} S_{0}^{l}}}{S_{0}^{0}} F_{p, q}\left[\begin{array}{ll}
c & d \\
a & b
\end{array}\right],
$$

where $i, j, k, l$ are the external legs of the block considered here. Our aim is to show that $W$ has the tetrahedral symmetry ${ }^{24}$. Let us introduce the following notation for the edges of a tetrahedron:

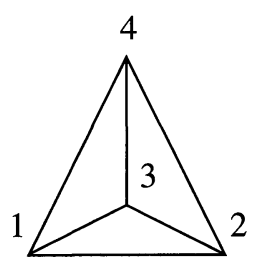

We know from Moore and Seiberg [14] that:

$$
\begin{aligned}
& F_{p, q}\left[\begin{array}{ll}
c & d \\
a & b
\end{array}\right]=F_{\hat{p}, q}\left[\begin{array}{ll}
\sigma_{23}(c) & \sigma_{12}(d) \\
\sigma_{13}(b) & \sigma_{13}(a)
\end{array}\right], \\
& F_{p, q}\left[\begin{array}{ll}
c & d \\
a & b
\end{array}\right]=F_{p, \hat{q}}\left[\begin{array}{ll}
\sigma_{23}(d) & \sigma_{23}(c) \\
\sigma_{23}(a) & \sigma_{12}(b)
\end{array}\right],
\end{aligned}
$$

which represent $W$ 's symmetry under (12) and (34) permutations. To go further, we start from the pentagonal identity $P_{23} F_{13} F_{12}=F_{23} F_{12} F_{23}$ acting on a block with external legs $\left\{, \hat{i}, j, k, l\right.$ and we project on the sector with $q_{1}=0$ :
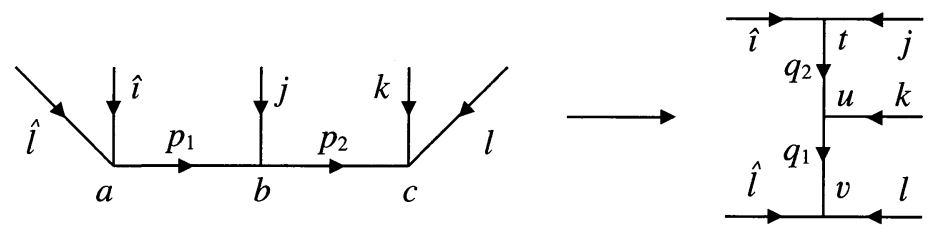

Using the fact that $F$ matrices with one external line set to zero act trivially gives us ${ }^{25}$ :

$$
F_{p_{2}, 0}\left[\begin{array}{cc}
t_{l} & t_{k} \\
x & c
\end{array}\right] F_{p_{1}, \hat{k}}\left[\begin{array}{cc}
x & t \\
a & b
\end{array}\right]=F_{p_{1}, 0}\left[\begin{array}{cc}
t_{l} & t_{i} \\
a & \alpha
\end{array}\right] F_{p_{2}, i}\left[\begin{array}{ll}
\alpha & t \\
b & c
\end{array}\right] .
$$

We know that $F_{p, 0}\left[\begin{array}{cc}t_{i} & t_{j} \\ a & b\end{array}\right]=0$ when $a \neq \hat{b}$. We shall now fix a gauge in such a

\footnotetext{
${ }^{24}$ In RTFT, it will become the expectation value of a tetrahedron

${ }^{25}$ In the rest of this appendix, we shall be sloppy about the vertex indices, denoting by $a$ the vertex $\sigma(a)$
} 
way that:

$$
F_{p, 0}\left[\begin{array}{cc}
t_{i} & t_{j} \\
a & b
\end{array}\right]=\delta_{a, \hat{b}} \sqrt{\frac{S_{0}^{0} S_{0}^{p}}{S_{0}^{i} S_{0}^{j}}}
$$

while preserving the $S$ matrix and the value of $F$ matrices an external line of which is set to 0. Reporting Eq. (51) in (50) gives $W$ 's symmetry under the 3-cycle (124). But is is clear that the group generated by (12), (34), and (124) is the full permutation group $\Sigma_{4}$ and therefore $W$ has the full tetrahedral symmetry.

Let us show that we can perform the previously mentioned gauge choice. We recall that Moore and Seiberg's equations are invariant under a rescaling of chiralvertex operators:

$$
\begin{aligned}
& F_{p, q}^{\prime}\left[\begin{array}{ll}
c & d \\
a & b
\end{array}\right]=\frac{\lambda_{a} \lambda_{b}}{\lambda_{c} \lambda_{d}} F_{p, q}\left[\begin{array}{ll}
c & d \\
a & b
\end{array}\right] \\
& B_{p, q}^{\prime}( \pm)\left[\begin{array}{ll}
c & d \\
a & b
\end{array}\right]=\frac{\lambda_{a} \lambda_{b}}{\lambda_{c} \lambda_{d}} B_{p, q}( \pm)\left[\begin{array}{ll}
c & d \\
a & b
\end{array}\right] \\
& S^{\prime}(j)_{(i, a)}^{\left(i^{\prime}, a^{\prime}\right)}=\frac{\lambda_{a}}{\lambda_{a^{\prime}}} S(j)_{(i, a)}^{\left(i^{\prime}, a^{\prime}\right)} \\
& T^{\prime}=T
\end{aligned}
$$

under the $a \mapsto \lambda_{a} \in \mathbf{C}^{*}$ gauge transformation. Preserving $S(0)$ implies that $\lambda_{t_{i}}=\lambda_{t_{0}}$. We fix $\lambda_{t_{0}}$ to be 1 by convention. Then, if $F\left\{\begin{array}{ll}j & k \\ i & l\end{array}\right\}$ is the $F$ matrix acting on blocks with external legs set to $i, j, k$ and $l$, we see that:

$$
F^{\prime}\left\{\begin{array}{ll}
i & j \\
0 & k
\end{array}\right\}=F\left\{\begin{array}{ll}
i & j \\
0 & k
\end{array}\right\} \text {. }
$$

The only non-zero entries of $F_{p, 0}\left\{\begin{array}{ll}j & \hat{j} \\ i & \hat{i}\end{array}\right\}$ are the $F_{p, 0}\left[\begin{array}{cc}t_{i} & t_{j} \\ a & \hat{b}\end{array}\right]$. We can therefore choose the $\lambda_{a} \mathrm{~s}$ in such a way that

$$
\lambda_{a} \lambda_{\hat{a}} F_{p, 0}\left[\begin{array}{cc}
t_{i} & t_{j} \\
a & \hat{a}
\end{array}\right]=\sqrt{\frac{S_{0}^{0} S_{0}^{p}}{S_{0}^{i} S_{0}^{j}}}
$$

\section{A.2. Unitarity of $F$ and $B$ Matrices}

We prove that the $F$ matrix is unitary, that is to say that:

$$
\sum_{q, x, y} F_{p, q}\left[\begin{array}{cc}
x & y \\
a & b
\end{array}\right] F_{p^{\prime}, q}\left[\begin{array}{cc}
x & y \\
a^{\prime} & b^{\prime}
\end{array}\right]^{*}=\delta_{a, a^{\prime}} \delta_{b, b^{\prime}} \delta_{p, p^{\prime}}
$$

From the relation between $F$ and $B$ and the hexagon, one obtains

$$
F B(\varepsilon)=(1 \otimes \Omega(-\varepsilon)) F
$$

from which $B(\varepsilon) B(-\varepsilon)=1$ and by replacing $B(\varepsilon)$ by $(\Omega(-\varepsilon) \otimes 1) F(1 \otimes \Omega(\varepsilon))$ in this identity, we find:

$$
\sum_{c, d} F_{p, q}\left[\begin{array}{ll}
c & d \\
a & b
\end{array}\right] F_{\hat{q}, \hat{p}^{\prime}}\left[\begin{array}{ll}
a^{\prime} & b^{\prime} \\
c & d
\end{array}\right]=\delta_{a, a^{\prime}} \delta_{b, b^{\prime}} \delta_{p, p^{\prime}},
$$


and using the tetrahedral symmetry

we obtain (56).

$$
F_{p, q}\left[\begin{array}{ll}
c & d \\
a & b
\end{array}\right]=F_{q, \hat{p}}\left[\begin{array}{ll}
\hat{b} & \hat{a} \\
\hat{c} & \hat{d}
\end{array}\right]=F_{\hat{q}, p}\left[\begin{array}{ll}
b & a \\
c & d
\end{array}\right]^{*},
$$

The relation between $F$ and $B$ together with $\Omega(\varepsilon)^{\dagger}=\Omega(\varepsilon)^{-1}=\Omega(-\varepsilon)$ imply $B(\varepsilon)^{\text {'s }}$ unitarity. Hence:

Lemma 10. The $F$ and $B( \pm)$ matrices acting on $H_{0,4}$ are unitary.

\section{B. Graph Invariants in $S_{3}$}

In this appendix, we shall recall how to define topological invariants for framed trivalent colored diagrams embedded into the sphere $S_{3}$. We shall firstly recall how to prove that we define topological invariants in $S_{3}$. For this purpose, we adapt a method used by Reshetikhin and Turaev in the case of Quantum Groups [24]. Then, we prove some useful properties of these invariants, namely, partial projective functoriality in $S_{3}$.

\section{B.1. Topological Invariance in $S_{3}$}

We define topological invariants in $S_{3}$ as explained in Subsect. 2.2. The basic idea is to use a transfer matrix method. Transfer matrices are defined in Subsect. 2.2. The partition function of a trivalent graph with normal framing is defined by Definition 6. The case of another framing introduces some phase factors described in Subsect. 2.2. Indeed, we want to prove Lemma 1: the number associated with a decomposition of the three sphere into cylinders sandwiched between two threeballs does not depend on the decomposition but only on $S_{3}$ 's decoration.

In a recent work, Reshetikhin and Turaev [24] explained how to generalize Reidemeister moves to the case of a framed trivalent graph in $S_{3}$. We shall firstly consider the case of graphs with a normal framing, also called homogeneous graphs. In this case, one can forget about it and say that $K$ and $K^{\prime}$ are equivalent if and only if there exists an isotopy which sends $K$ onto $K^{\prime}$ and which is compatible with the colorings of the graphs. That is to say, a line (a vertex respectively) is brought on a line (a vertex respectively) with the same color. Following the terminology of [24], we only consider homogeneous colored directed ribbon graphs (HCDR). The HCDR category is defined in [24]. It is generated by some particular graphs as explained in [24]. These generators obey some relations as expected from the case of links and knots $^{26}$. In [24], a complete set of relations is given for generators of the category HCDR. We recall these relations in Fig. 10. We have to check that our assignment of operators with these generators is consistent with these relations. This will prove that we have defined a functor from the category HCDR into a category $\mathbf{S p}^{\prime}$ defined as follows: its objects are the spaces $^{27} H_{0, n}^{[E]}$ for $n \in \mathbf{N}$ and its morphisms are linear operators between these spaces. The composition of morphisms is the composition of linear operators. We define $\mathbf{F}$ by its action on morphisms and objects as follows:

Action on Objects. As explained in [24], HCDR's objects are finite sequences of distinct points in $\mathbf{R}^{2}$ labelled with line indices and signs $\left[\left(P_{k}, j_{k}, \varepsilon_{k}\right)_{k}\right]$. The empty set

${ }^{26}$ The reader will recall the celebrated Reidemeister moves

27 We recall that $[E]$ denotes a coloring of the external lines which are assumed to be incoming 


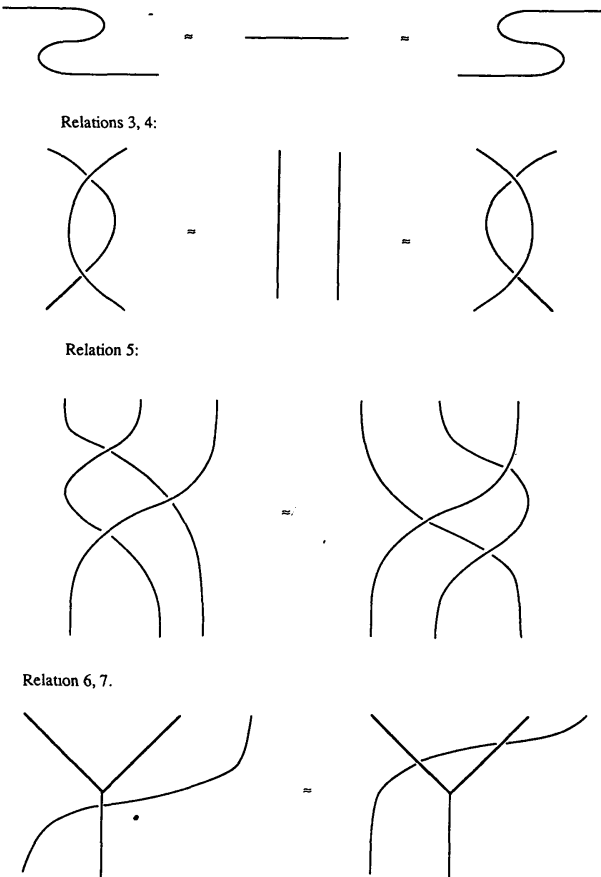

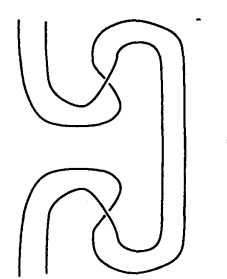

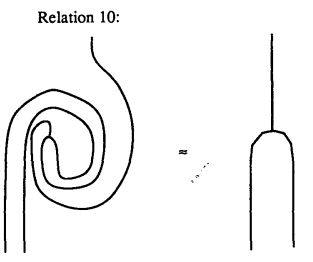

Relation 12:

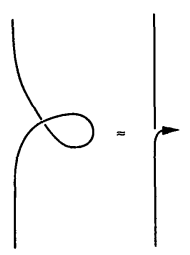

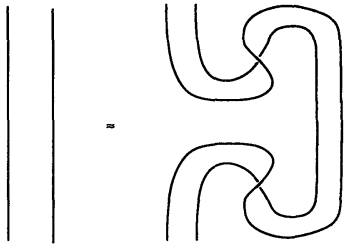

Relation 11:

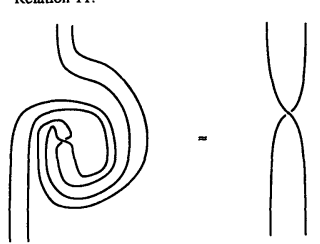

Relation 13:

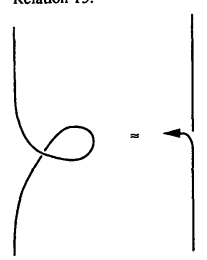

Fig. 10. Defining relations of category HCDR. Relations 1 to 5, 8, 9, and 11 are called Reidemeister relations and can be used to define knot and link invariants. Relation 5 is the celebrated YangBaxter relation. All other relations involve vertices. Relations 12 and 13 describe compatibility between crossings and changes of framings. On our figure, the arrow describes the sense in which the framing is turning. It is supposed to be normal, pointing towards the reader before the arrow which indicates how it rotates around the line we consider. Beware that on all our drawings lines have not been oriented because any change of orientation can be obtained by turning a coloring into its opposite. Remember also that wherever a crossing appears, a similar relation holds exchanging over- and under-crossings. Invariance of Moore and Seiberg's equations under permutation of $B(+)$ and $B(-)$ makes their considering irrelevant

$\emptyset$ is also considered as an object. Let $n$ be the cardinal of the set considered here, $\mathbf{F}$ associate with it $H_{0, n}^{\left[\left(l_{k}\right)_{k}\right]}$, where $l_{k}=j_{k}$ or $\hat{\jmath}_{k}$ according to $\varepsilon_{k}=1$ or -1 .

Action on Morphisms. HCDR's morphisms consists into trivalent graphs included in $\mathbf{R}^{2} \times[0,1]$ interpolating between two objects located in boundary planes of $\mathbf{R}^{2} \times[0,1]$. $\mathscr{G}^{\prime}$ 's coloring should be compatible with the boundary indices. More precisely, the color of a line arriving at point $P_{k}$ is $j_{k}$ and the line is oriented accordingly to $\varepsilon_{k}$. For example, when $\varepsilon_{k}=1$, the line has to be oriented by a descending arrow. Given such a morphism, we decompose it into generators $A_{k}$ as explained before and the transfer matrix computation enables us to define $\mathbf{F}[\mathscr{G}]$ by:

$$
\mathbf{F}[\mathscr{G}]=\prod_{k=0}^{m} \hat{A}_{m-k}, \quad\left[\mathbf{R}^{2} \times[0,1], \mathscr{G}\right]=\#_{k \in\langle 0, m\rangle} A_{k} .
$$

Among all relations between generators, we can separate Reidemeister relations which involve no vertex and the other ones. This distinction is already made on Fig. 10. It is quite easy to check that Reidemeister relations are satisfied. Indeed, relation 3 is implied by Yang-Baxter's equation on $B( \pm)$ which is a 
consequence of Moore and Seiberg's equations as was shown in [14]. Let us sketch a proof of the other relations.

Relations 6,7. We slide a line across a vertex. Relation

$$
F_{13} B_{12}( \pm)=B_{13}( \pm) B_{12}( \pm) F_{23}
$$

proves relations 6 and 7. This relation is a consequence of Moore and Seiberg's equations obtained by combining the pentagon and the relation between $F$ and $B( \pm)$. We also have to use the tetrahedral symmetry of $F$.

Relations 8.9. We proceed in two steps. First of all we prove the identities illustrated on Fig. 11. We use, one more time, the tetrahedral symmetry of $F$. Then, from $B(+) B(-)=1$, we deduce relations 8 and 9 .

Relations 10,11 . These identities can be checked quite trivially and we do not need really any deep Moore and Seiberg identity.

Relation 12,13. These identities do not appear under this form in the work of Reshetikhin and Turaev [24] but our identities imply relation 8 of their paper. Moreover, relations 12 and 13 shows that twisting the framing of a line carrying index $j$ introduces a phase factor $\exp \left(2 \pi i h_{j}\right)$. Obviously, the left-hand side of our relations are topologically equivalent to twistings of line's framings by \pm 1 unit.

To prove this relation, let us compute in the transfer matrix formalism the lefthand side of relation 12 as on Fig. 12. This gives:

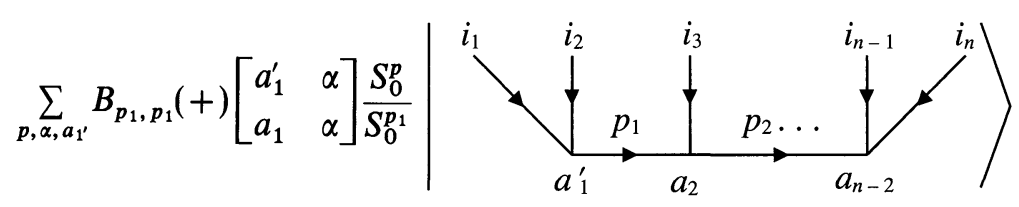
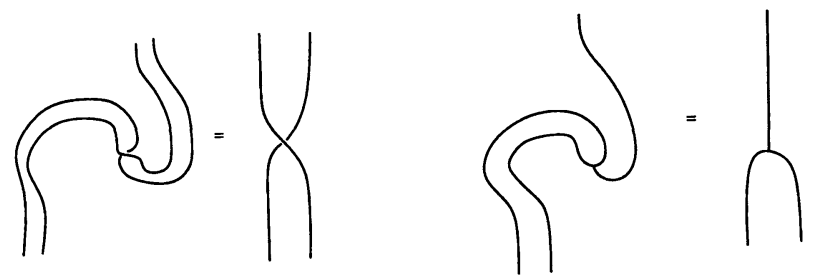

Fig. 11. Intermediate relations needed to prove relations 8 and 9

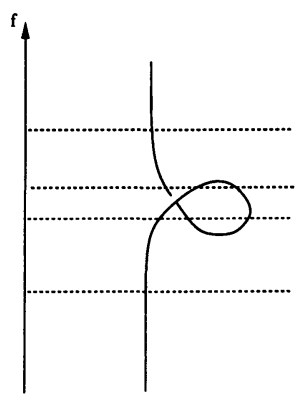

Fig. 12. Morse description of the left-hand side of relation 12 
Note that $\alpha$ 's external legs are $p_{1}, i_{2}, p$. The extra-hypotheses we made on $F$ and our suitable gauge choice together with the relation between $F$ and $B$ provide us with:

$$
B_{0, p}(+)\left[\begin{array}{cc}
\alpha & \beta \\
t_{p} & t_{i_{2}}
\end{array}\right]=\sqrt{\frac{S_{0}^{0} S_{0}^{p}}{S_{0}^{i_{2}} S_{0}^{p_{1}}}} \exp \left(\pi i\left(h_{i_{2}}+h_{p_{1}}-h_{p}\right)\right) \delta_{\alpha, \hat{\beta}}
$$

and reporting this into (58) gives:

$$
\begin{aligned}
& \frac{S_{0}^{i_{2}}}{S_{0}^{0}} \sum_{p, m, x, y} B_{p_{1}, m}(+)\left[\begin{array}{ll}
a_{1}^{\prime} & y \\
a_{1} & \alpha
\end{array}\right] B_{p, q}(+)\left[\begin{array}{cc}
\alpha & \alpha \\
t_{p_{1}} & t_{i_{2}}
\end{array}\right] B_{p, 0}(+)\left[\begin{array}{cc}
t_{p_{1}} & t_{i_{2}} \\
y & \hat{\alpha}
\end{array}\right]
\end{aligned}
$$

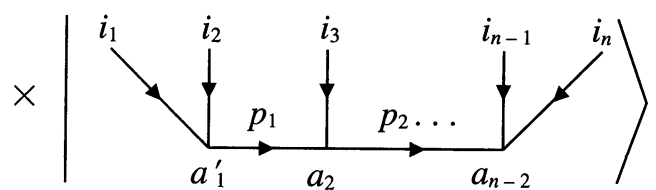

However, let us stress that:

$$
B_{p_{1}, m}(+)\left[\begin{array}{ll}
a_{1}^{\prime} & y \\
a_{1} & \alpha
\end{array}\right] B_{p, q}(+)\left[\begin{array}{cc}
\alpha & \alpha \\
t_{p_{1}} & t_{i_{2}}
\end{array}\right] B_{p, 0}(+)\left[\begin{array}{cc}
t_{p_{1}} & t_{i_{2}} \\
y & \hat{\alpha}
\end{array}\right]
$$

is nothing but the matrix element of $B_{23}(+) B_{12}(+) B_{23}(+)$ between the states:

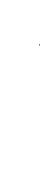

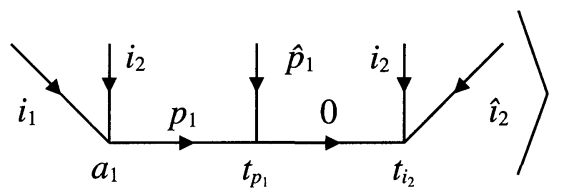

and

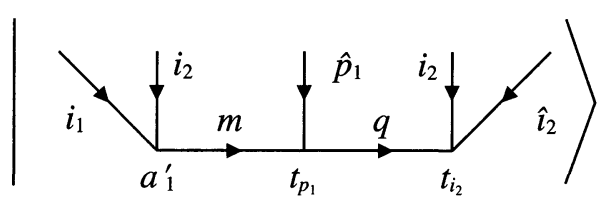

and we shall now use Yang-Baxter's equation. The matrix element of

$$
B_{12}(+) B_{23}(+) B_{12}(+)
$$

between the states we considered is:

$$
B_{p_{1}, i_{2}}(+)\left[\begin{array}{cc}
x & t_{i_{2}} \\
a_{1} & t_{p_{1}}
\end{array}\right] B_{0,0}(+)\left[\begin{array}{cc}
t_{i_{2}} & t_{i_{2}} \\
t_{i_{2}} & t_{i_{2}}
\end{array}\right] B_{i_{2}, p_{1}}(+)\left[\begin{array}{cc}
a_{1}^{\prime} & t_{p_{1}} \\
x & t_{i_{2}}
\end{array}\right] .
$$

It can trivially be computed and we find:

$$
\frac{S_{0}^{0}}{S_{0}^{i}} \exp \left(2 \pi i\left(h_{i_{1}}-h_{p_{1}}\right)\right)
$$

Finally, all this shows that:

$$
\sum_{\alpha, p} \frac{S_{0}^{p}}{S_{0}^{p_{1}}} B_{p_{1}, p_{1}}(-)\left[\begin{array}{cc}
x & \alpha \\
a_{1} & \alpha
\end{array}\right]=\delta_{x, a_{1}} \exp \left(-2 i \pi h_{i_{2}}\right),
$$

and performing the same computation with an undercrossing produces the opposite phase. This proves our relations.

This completes our analysis in the case of homogeneous graphs. Let us consider the case when the framing is not normal. A twist in the framing of a line carrying the index $j$ by $n$ units multiplies the partition function by $\exp \left(2 \pi i n h_{j}\right)$. When proving relations 8 and 9 , we have seen that this interpretation was indeed consistent with isotopies in $S_{3}$ because we can always untwist the framing of a line by introducing a suitable crossing as on relation 12 . Finally, our prescription defines topological invariants for framed colored $\phi^{3}$ diagrams in $S_{3}$. 


\section{B.2. Properties of these Invariants}

First of all, we study the behaviour of these invariants under an orientation reversing symmetry and a reversing of the coloring. Then we show that they satisfy functoriality when $S_{3}$ is cut along a two-sphere $S_{2}$ and projective functoriality when one uses a genus $g$ surface with an arbitrary number of punctures embedded in a trivial way. We shall also prove that any line carrying an index 0 can be deleted.

B.2.1. Changing the Orientation of the Graph. Let us start from a framed graph $K$ colored by $\mathscr{K}$. We define $\widetilde{K}$ to be the graph obtained from $K$ through an orientation reversing symmetry ${ }^{28}$. The framing itself is affected by this symmetry and turned into its opposite. We claim that:

Lemma 11. With the above definitions:

$$
Z\left[S_{3}, \tilde{K}_{\hat{K}}\right]=Z\left[S_{3}, K_{\mathscr{K}}\right]^{*} .
$$

Proof. We compute $Z\left[S_{3}, K_{\mathscr{K}}\right]$ using a projection plane $\Pi$. First of all, using isotopy, $K$ 's framing can be chosen to be normal. Henceforth $\widetilde{K}$ is obtained from $K$ by symmetry through $\Pi$. It has normal framing. Its projection on $\Pi$ differs from $K$ 's one by turning over (respectively under) crossings into under (respectively over) crossings.

From Eq. (10) and the relation between $F$ and $B$ matrices, we find:

$$
B_{\hat{p}, \hat{q}}(-\varepsilon)\left[\begin{array}{ll}
\hat{c} & \hat{d} \\
\hat{a} & \hat{b}
\end{array}\right]=\left(B_{p, q}(\varepsilon)\left[\begin{array}{ll}
c & d \\
a & b
\end{array}\right]\right)^{*} \text {. }
$$

Moreover, a famous expression of $S$ in terms of $B$ and $F$ matrices can be inferred from Moore and Seiberg's equations:

$$
\frac{S_{i}^{j}}{S_{0}^{0}}=\frac{(B(-) B(-))_{0,0}\left[\begin{array}{ll}
t_{i} & t_{j} \\
t_{i} & t_{j}
\end{array}\right]}{F_{i} F_{j}}, \quad F_{i}=F_{0,0}\left[\begin{array}{cc}
t_{i} & t_{i} \\
t_{i} & t_{i}
\end{array}\right]
$$

and it shows that $S_{i}^{\hat{j}}=\left(S_{i}^{j}\right)^{*}$. In particular $S_{0}^{\hat{\imath}}=S_{0}^{i}$ and is real. Therefore, through the use of the transfer matrix method, it is obvious that:

$$
Z\left[S_{3}, \tilde{K}_{\hat{\mathscr{K}}}\right]=Z\left[S_{3}, K_{\mathscr{K}}\right] \text {. }
$$

B.2.2. Functoriality along $S_{2}$. We consider $K \subset S_{3}$ a trivalent graph, framed and colored in $S_{3}$. We are given $\Sigma_{0, n}$ a two sphere embedded in $S_{3}{ }^{29}$. We assume that $K$ is cut into two pieces $K_{ \pm}$by this surface and no vertex belongs to $K_{+} \cap K_{-}$. Therefore, we can decompose $\left[S_{3}, K\right]$ into cylinders in order to compute $Z\left[S_{3}, K\right]$ in such a way that $\Sigma_{0, n}$ is the frontier of one of the cylinders. The graph $\mathscr{G}$ we use is the so-called multiperipheral graph and using suitable Morse decompositions for $\mathscr{G}$ to compute $Z\left[S_{3}, \mathscr{G}_{\hat{\mathscr{C}}} \# K_{+}\right]$shows that:

$$
Z\left[S_{3}, K\right]=\sum_{\mathscr{C}} \frac{Z\left[S_{3}, \mathscr{G}_{\mathscr{C}} \# K_{+}\right] Z\left[S_{3}, K_{-} \# \mathscr{G}_{\hat{\mathscr{C}}}\right]}{\left\langle B_{3}, \mathscr{G}_{\mathscr{C}} \mid B_{3}, \mathscr{G}_{\mathscr{C}}\right\rangle} .
$$

Amusingly, this is nothing but functoriality in this particular case.

${ }^{28}$ Such as a plane symmetry, no matter which plane we choose thanks to topological invariance

${ }^{29} n$ is the cardinal of $K \cap \Sigma_{0, n}$ 
B.2.3. Other Bases of $H_{0, n}$. Let us show that it is possible to build many useful bases of $H_{0, n}$ for $n \geqq 4$. These bases are related by duality transformations (i.e. by the $F$ and $B$ matrices) and will be used in the proof of functoriality for $g \geqq 1$.

Let us be given a genus zero $\phi^{3}$ diagram $\mathscr{G}$ with $n$ external legs. Any other genus 0 trivalent graph with $n$ labelled external legs $\mathscr{G}^{\prime}$ is related to $\mathscr{G}$ by a finite sequence of $F$ and $B$ moves defined as in Subsect. 2.1.

As in Subsect. 4.1, we shall see that our formalism is covariant. $P\left(\mathscr{G}, \mathscr{G}^{\prime}\right)$ is defined as in Subsect. 2.1. We prove that formula 64 holds with any graph $\mathscr{G}$ by recurrence.

Let us assume that formula (64) holds for a given graph $\mathscr{G}$. We shall prove that it holds for any graph $\mathscr{G}^{\prime}$ related to $\mathscr{G}$ by an elementary move $F$ or $B( \pm)$. Then, finiteness of the duality complex implies that the formula is true for any tree-level graph $\mathscr{G}$ with $n$ external legs.

To perform the recurrence step, we consider $\mathscr{G}$ and $\mathscr{G}^{\prime}$ differing by an $F$-move. ${ }^{30}$ We can find two three-balls $B_{ \pm}$in $S_{3}$ such that $\left(\mathscr{G}_{\hat{\mathscr{E}}} \# K_{+}\right) \cap B_{+}$and $\left(K_{-} \# \mathscr{G}_{\mathscr{G}}\right) \cap B_{-}$ look as on the following figure:
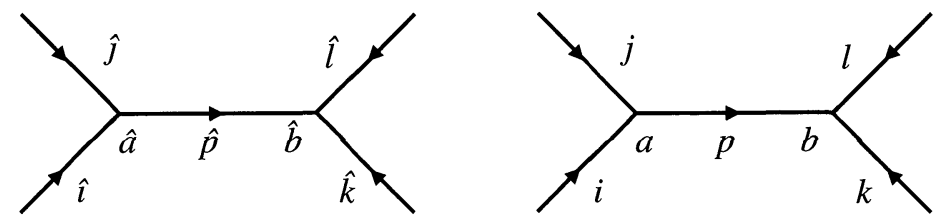

$$
\left(\mathscr{G}_{\dot{\mathscr{G}}} \# K_{+}\right) \cap B_{+} \quad\left(K_{-} \# \mathscr{G}_{\mathscr{C}}\right) \cap B_{-}
$$

and changing $\mathscr{G}$ into $\mathscr{G}^{\prime}$ is performed by replacing $B_{ \pm}$by the following three-balls:

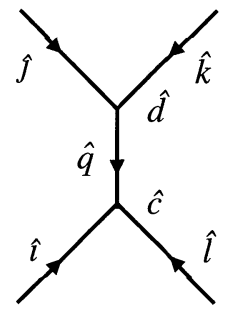

$\left(\mathscr{G}_{\dot{G}^{\prime}}^{\prime} \# K_{+}\right) \cap B_{+}$

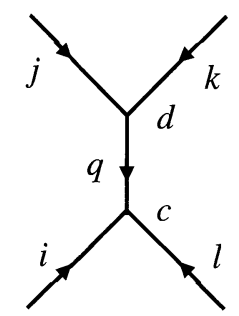

$\left(K_{-} \# \mathscr{G}_{\mathscr{G}^{\prime}}^{\prime}\right) \cap B_{-}$

Using the transfer matrix method and cutting along $B_{ \pm}$'s boundaries shows that:

$$
\begin{aligned}
& Z\left[S_{3}, \mathscr{G}_{\hat{\mathscr{C}}} \# K_{+}\right]=\sum_{c, d} F_{p, q}\left[\begin{array}{ll}
c & d \\
a & b
\end{array}\right]^{*} Z\left[S_{3}, \mathscr{G}_{\dot{\mathscr{C}}^{\prime}}^{\prime} \# K_{+}\right], \\
& Z\left[S_{3}, K_{-} \# \mathscr{G}_{\mathscr{C}}\right]=\sum_{c, d} F_{p, q}\left[\begin{array}{ll}
c & d \\
a & b
\end{array}\right] Z\left[S_{3}, K_{-} \# \mathscr{G}_{\mathscr{C}^{\prime}}^{\prime}\right],
\end{aligned}
$$

where $(p, a, b)$ and $(q, c, d)$ are contained in $\mathscr{C}$ and $\mathscr{C}^{\prime}$ respectively. Using $F$ 's unitarity, we see that:

$$
\begin{aligned}
Z\left[S_{3}, K\right] & =\sum_{\mathscr{C}} \frac{Z\left[S_{3}, \mathscr{G}_{\mathscr{C}} \# K_{+}\right] Z\left[S_{3}, K_{-} \# \mathscr{G}_{\mathscr{C}}\right]}{\left\langle B_{3}, \mathscr{G}_{\mathscr{C}} \mid B_{3}, \mathscr{G}_{\mathscr{C}}\right\rangle} \\
& =\sum_{\mathscr{C}^{\prime}} \frac{Z\left[S_{3}, \mathscr{G}_{\mathscr{C}^{\prime}}^{\prime} \# K_{+}\right] Z\left[S_{3}, K_{-} \# \mathscr{G}_{\mathscr{C}^{\prime}}^{\prime}\right]}{\left\langle B_{3}, \mathscr{G}_{\mathscr{C}^{\prime}}^{\prime} \mid B_{3}, \mathscr{G}_{\mathscr{C}^{\prime}}^{\prime}\right\rangle},
\end{aligned}
$$

30 The case of $B$-moves is treated in the same way 
and this proves formula (64) for $\mathscr{G}^{\prime}$ deduced from $\mathscr{G}$ by an $F$-move. Hence, this formula is true for any genus 0 graph $\mathscr{G}$, with $n$ external legs.

This proves the following lemma:

Lemma 12. Functoriality holds along a two-sphere in $S_{3}$ :

$$
Z\left[S_{3}, K\right]=\sum_{\mathscr{C}} \frac{Z\left[S_{3}, \mathscr{G}_{\mathscr{C}} \# K_{+}\right] Z\left[S_{3}, K_{-} \# \mathscr{G}_{\hat{\mathscr{C}}}\right]}{\left\langle B_{3}, \mathscr{G}_{\mathscr{C}} \mid B_{3}, \mathscr{G}_{\mathscr{C}}\right\rangle}
$$

for any tree-level graph $\mathscr{G}$ with $n$ external legs.

It is then obvious to infer from that the following result:

Lemma 13. Functoriality holds along a two-sphere in any closed three-manifold!

Proof. Let $M=\left[M_{1}, K_{1}\right] \#\left[M_{2}, K_{2}\right]$ be a three-manifold cut along a two-sphere. $K_{1} \# K_{2}$ denotes its decoration. As Lemma 7 shows us, $M$ can be obtained by surgery from $S_{3}$ along $\left[\left(L_{1}, L_{2}\right),\left(\bar{K}_{1}, \bar{K}_{2}\right)\right]$, where $\left(L_{1}, \bar{K}_{1}\right)$ and $\left(L_{2}, \bar{K}_{2}\right)$ are separated by a two-sphere $S_{2}$ which corresponds through the surgery to the one used to cut $M$. Functoriality along $S_{2}$ in $S_{3}$ makes clear that we only have to worry about phase factors.

Here comes the work of Atiyah [31] who showed that $\sigma_{L}$ is indeed proportional to the integral of the first Pontryagin class of the tangent bundle on $W_{L}$. Additivity properties of Pontryagin classes imply that $\sigma\left(L_{1} \cap L_{2}\right)=\sigma\left(L_{1}\right)+\sigma\left(L_{2}\right)$ when $L_{1}$ and $L_{2}$ can be separated by a two-sphere. Therefore we end up with functoriality along $S_{2}$ in any three-manifold:

$$
\begin{aligned}
& Z\left[M_{1} \# B_{3}, K_{1} \# \mathscr{G}_{\mathscr{C}}\right]=e^{-2 \pi i c \sigma\left(L_{1}\right) / 8} \sum_{\mathscr{C}_{1}}\left(\prod_{k=1}^{n_{1}}\left(\left(e^{2 \pi i c / 24} T\right)^{-n_{k}} S\right)_{\mathscr{C}_{1, j}}\right) \\
& \times Z\left[S_{3}, \bar{K}_{1} \# \mathscr{G}_{\mathscr{C}}, L_{1, \mathscr{C}_{1}}\right] \text {, } \\
& Z\left[B_{3} \# M_{2}, \mathscr{G}_{\mathscr{C}} \# K_{2}\right]=e^{-2 \pi i c \sigma\left(L_{2}\right) / 8} \sum_{\mathscr{C}_{2}}\left(\prod_{k=1}^{n_{2}}\left(\left(e^{2 \pi i c / 24} T\right)^{-n_{k}} S\right)_{\mathscr{C}_{2, j}}\right) \\
& \times Z\left[S_{3}, \mathscr{G}_{\mathscr{C}} \# \bar{K}_{2}, L_{2, \mathscr{C}_{2}}\right], \\
& Z[M]=e^{-2 \pi i c \sigma\left(L_{1} \cup L_{2}\right) / 8} \sum_{\mathscr{C}_{1}, \mathscr{C}_{2}}\left(\prod_{k=1}^{n_{1}}\left(\left(e^{2 \pi i c / 24} T\right)^{-n_{k}} S\right)_{\mathscr{C}_{1, j}}\right) \\
& \times\left(\prod_{k=1}^{n_{2}}\left(\left(e^{2 \pi i c / 24} T\right)^{-n_{k}} S\right)_{\mathscr{C}_{2, j}}\right) Z\left[S_{3}, \bar{K}_{1} \# K_{2}, L_{1, \mathscr{C}_{1}}, L_{2, \mathscr{C}_{2}}\right] .
\end{aligned}
$$

B.2.4. Partial Projective Functoriality for $g \geqq 1$. Our strategy will be to prove formula (65) for $g=1$ and then prove it for any genus. Before going into details, let us examine precisely our problem.

Instead of $\Sigma_{0, n}$, we consider $\Sigma_{g, n}$ a genus $g$ surface embedded in $S_{3}$ which intersects $K$ in $n$ points, none of which is a vertex. This surface splits $S_{3}$ into two connected components. We assume that one of these components can be viewed as a genus $g$ handlebody $T_{g}$ and the other one as $S_{3} \backslash T_{g}$. Let us however stress that an embedding of $\Sigma_{g}$ in $S_{3}$ does not necessarily split it into a handlebody and its complementary which is diffeomorphic to a handlebody. This is why we only prove a partial projective functoriality theorem which is the only thing we need in Subsect. 4.3.3. 
The property we want to prove is the following:

$$
Z\left[M_{-} \# M_{+}\right]=e^{2 \pi i c \sigma_{g} / 12} \sum_{\mathscr{C}} \frac{Z\left[S_{3}, \mathscr{G}_{\mathscr{C}} \# K_{+}\right] Z\left[T_{g} \# \hat{T}_{g}, K_{-} \# \mathscr{G}_{\hat{\mathscr{C}}}\right]}{\left.\| T_{g}, \mathscr{G}_{\mathscr{C}}\right\rangle \|^{2}} .
$$

$Z\left[S_{3}, \mathscr{G}_{\mathscr{C}} \# K_{+}\right]$is a partition function in $S_{3}$ and can be computed using the transfer matrix method. On the other hand, $Z\left[T_{g} \# \hat{T}_{g}, K_{-} \# \mathscr{G}_{\hat{\mathscr{E}}}\right]$ must be computed using a surgery presentation of $T_{g} \# \widehat{T}_{g}$. Let us now prove (65), first in genus one, then in any genus.

Case of Genus One. We shall here study the case $g=n=1$. In this case, we can homotopically transform $K_{ \pm}$as indicated on Fig. 13. This singularizes the homotopically non-trivial lines of $K_{-}$in $T_{1}$ and of $K_{+}$in $S_{3} \backslash T_{1}$. We shall make an intensive use of the previous lemma and of topological invariance of all partition functions. This result is proved in Sect. 3.2 without using more than functoriality along $S_{2}$ in $S_{3}$ ! Therefore, it can be used safely.

For the sake of simplicity, we shall firstly consider the case depicted on Fig. 13 where only one line is circulating in the loop of $T_{1}\left(S_{3} \backslash T_{1}\right.$ respectively). The usual basis of $H_{1,1}$ can be denoted as $\left(\left|\chi_{i, a}^{j}\right\rangle\right)_{i, j, a}$ where $a$ labels the vertex, $i$ the loop-line and $j$ the incoming external line. Let us compute $Z\left[M_{+} \#\left(\hat{T}_{1}, \chi_{k, b}^{j}\right)\right]$ and $Z\left[\left(T_{1}, \chi_{\hat{k}, \hat{b}}^{\hat{b}}\right) \# M_{-}\right]$.

- The first partition function gives:

$$
Z\left[M_{+} \#\left(\hat{T}_{1}, \chi_{\hat{k}, \hat{b}}^{\hat{b}}\right)\right]=\delta_{j, \hat{p}^{\prime}} \frac{1}{S_{0}^{j}} Z\left[S_{3}, \hat{k}^{a^{\prime}} i^{j} i^{\prime}\right\} Z\left[S_{3}, \mathscr{L}_{+}\right],
$$

where $\mathscr{L}_{+}$is the graph depicted on Fig. 14. To obtain this, we have cut along a twopunctured sphere. This operation is allowed thanks to the preceding result.

- Gluing two genus one handlebodies produces $S_{2} \times S_{1}$ which can be obtained from $S_{3}$ by surgery along an unknotted circle of framing zero. It is an easy exercise to check that $\mathscr{G}_{l}$ depicted on Fig. 15 naturally arises as the antecedent of the graph lying in $S_{2} \times S_{1}$. The partition function is then:

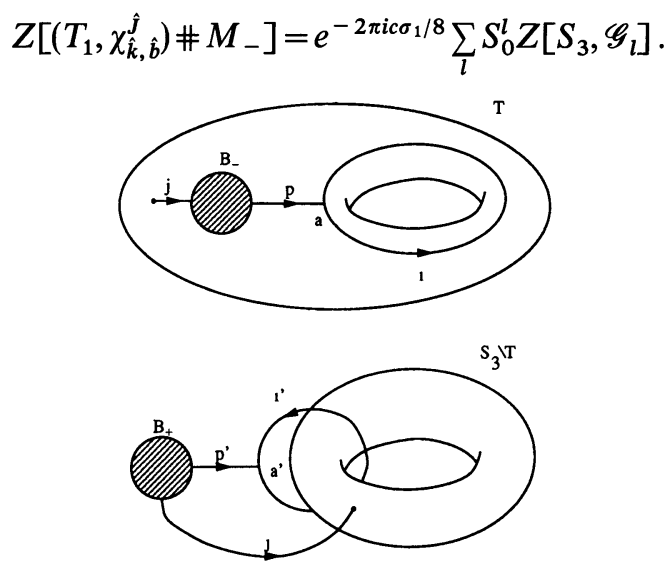

Fig. 13. Graphs $K_{+} \subset S_{3} \backslash T_{1}$ and $K_{-} \subset T_{1} . B_{ \pm}$contains their homotopically trivial parts 


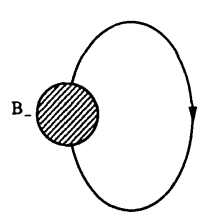

$\mathrm{B}+$

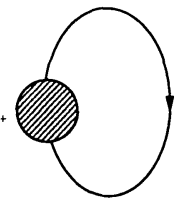

Fig. 14. Auxiliary graphs $\mathscr{L}_{+}$and $\mathscr{L}_{-}$; we have $p^{\prime}=\hat{j}$ and $p=j$

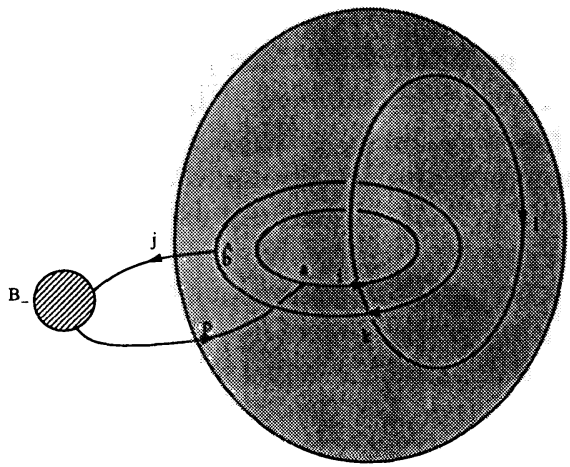

Fig. 15. Graph $\left(K_{+} \# \mathscr{G}_{\mathscr{C}}\right) \subset S_{3}$ : the sphere along which we cut is slightly shadowed

Using Lemma 13 we get after a short computation:

$$
Z\left[\left(T_{1}, \chi_{k, b}^{j}\right) \# M_{-}\right]=\frac{\delta_{j, p} e^{-2 \pi i c \sigma_{1} / 8}}{S_{0}^{j}} Z\left[S_{3}, \mathscr{L}_{-}\right]\left\langle\chi_{i, a}^{j} \mid \chi_{k, b}^{j}\right\rangle
$$

and $\mathscr{L}_{-}$is depicted on Fig. 14.

Putting Eqs. (66) and (67) together to compute $\left\langle M_{+} \mid M_{-}\right\rangle$produces precisely

$$
Z\left[S_{3}, K\right] \times e^{-2 \pi i c \sigma_{1} / 8} \text {. }
$$

This is easily proved by cutting $S_{3}$ along a two-puncture sphere to isolate the "bulbs" of $K_{+}$and $K_{-}$from the rest. Cutting again along a two-punctured sphere separates these bulbs, thus leading to the result.

Let us have a look at the more complicated case where several lines run into the non-trivial loops of $T_{1}$ and $S_{3} \backslash T_{1}$. The idea is to go back to the preceding case by cutting along suitable spheres to get rid of these lines. Let us consider $Z\left[M_{+} \#\left(\hat{T}_{1}, \chi_{k, b}^{j}\right)\right]$ for example. We can find a $2 m$-punctured sphere which contains the $m$ homotopically non-trivial lines of $M_{+}$as indicated on Fig. 16 . We then use a suitable basis of $H_{0,2 m}$ introduced in Subsect. 3.2 to compute $Z\left[M_{+} \#\left(\hat{T}_{1}, \chi_{k, b}^{j}\right)\right]$ by cutting along the $2 m$-punctured sphere. Consequently, this partition function is a linear combination of partition functions in $S_{3}$, the coefficients of which are independent of $(j, k, b)$. The same manipulation can be performed on $Z\left[\left(T_{1}, \chi_{\hat{k}, \hat{b}}^{\hat{b}}\right) \# M_{-}\right]$because these manipulations commute with the surgery used to pass from $S_{3}$ to $T_{1} \# \hat{T}_{1}$. Finally, we are back to the case where only one line is running in the homotopically non-trivial loops of $T_{1}$ and $S_{3} \backslash T_{1}$. These show that $\left\langle M_{+} \mid M_{-}\right\rangle$is expressed as a double linear combination of partition functions in $S_{3}$. But, the coefficients that appear here can be recovered from $Z\left[S_{3}, K\right]$ by cutting along punctured spheres that contain the homotopically non-trivial lines in $M_{ \pm}$. Hence, this proves formula (65) in this case. 


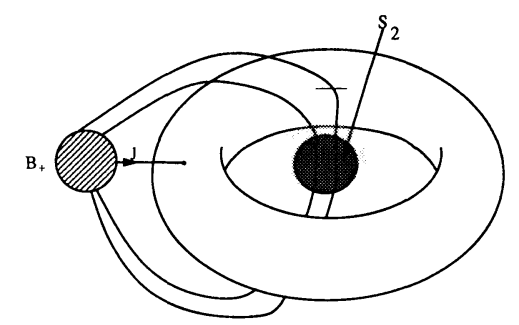

Fig. 16. By cutting along the $2 m$-punctured sphere $S_{2}$, we go back to the case where one line is running along the non-trivial loop of $S_{3} \backslash T_{1}$. The sphere along which we cut is slightly shadowed

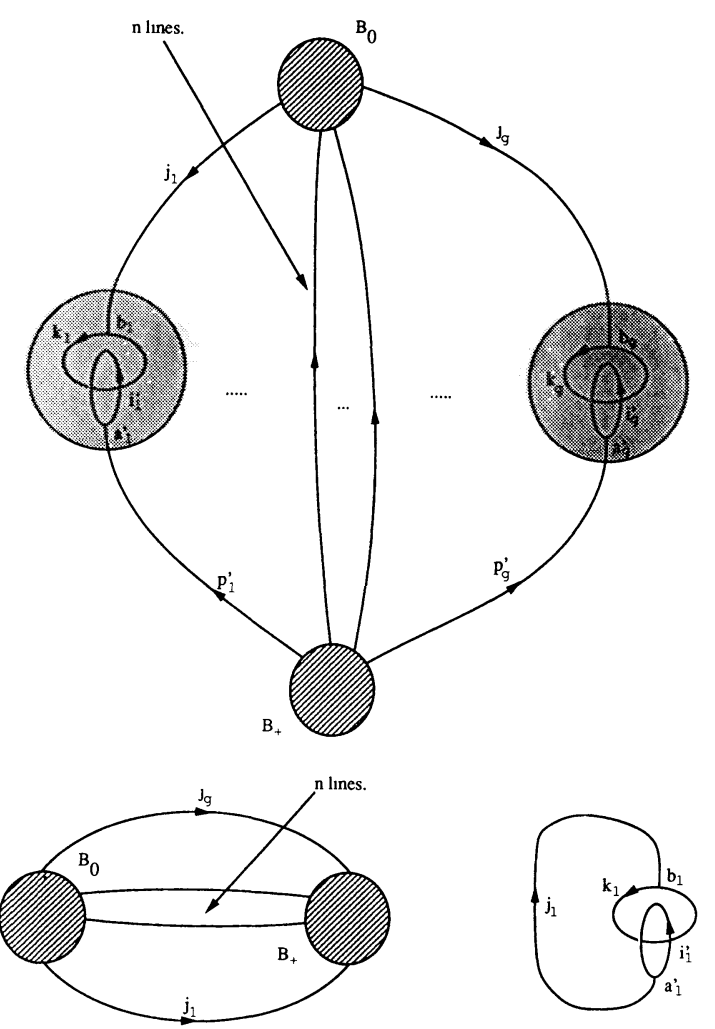

Fig. 17. We show $\left(K_{+} \# \mathscr{G}_{\mathscr{C}}\right) \subset S_{3}$ and the auxiliary graphs obtained by cutting along punctured spheres in $S_{3}$. These spheres are slightly shadowed

Lemma. Projective partial functoriality along a one-punctured torus holds in $S_{3}$.

Higher Genus and $g=1, n \geqq 2$. The result simply follows from the previous lemma and suitable splittings of the manifolds we consider.

We first look at the case where only one line runs along each non trivial cycle of $M_{ \pm}$. We choose a particular graph $\mathscr{G}$ which is shown on Fig. 17. The main restriction on its shape is that the line running along a non-trivial loop in $T_{g}$ has only one vertex. On this figure, $B_{0}$ contains the connection between the loops and the external legs of $\mathscr{G}$, that is to say, a tree with $n+g$ external legs. $Z\left[M_{+} \#\left(\hat{T}_{g}, \mathscr{G}_{\mathscr{C}}\right)\right]$ 
is a partition function in $S_{3}$. We then cut along suitable two-spheres to isolate the linked lines $i_{l}^{\prime}, k_{l}$ (see Fig. 17). This gives us:

$$
Z\left[M_{+} \#\left(\hat{T}_{g}, \mathscr{G}_{\mathscr{C}}\right)\right]=Z\left[S_{3}, \mathscr{K}_{\mathscr{C}}^{+}\right] \prod_{l=1}^{g} \frac{\delta_{j i}, \hat{p}_{l}}{S_{0}^{j i}} Z\left[S_{3}, \chi_{k_{l}, \alpha_{l}}^{p_{l}} \# \chi_{i^{\prime} \hat{l}, a_{l}}^{j_{i}^{\prime}}\right]
$$

where $\mathscr{K}_{\mathscr{C}}^{+}$is shown on Fig. 17 and $\chi_{k_{l}, \alpha_{l}}^{p_{l}}$ and $\chi_{i^{\prime} l, a_{l}^{\prime}}^{j_{l}}$ are linked as indicated on the same figure.

Using functoriality along $S_{2}$ in any three-manifold (Lemma 13), we cut $T_{g} \# \widehat{T}_{g}$ as indicated on Fig. 18 and this gives:

$$
Z\left[T_{g} \# \widehat{T}_{g}, \mathscr{G}_{\hat{\mathscr{C}}} \# K_{-}\right]=Z\left[S_{3}, \mathscr{K}_{\hat{\mathscr{C}}}^{-}\right] \prod_{l=1}^{g} \frac{\delta_{p_{l}, j_{l}}}{S_{0}^{j_{l}}} Z\left[T_{1} \# \hat{T}_{1}, \chi_{\hat{k}_{l}, \hat{\alpha}_{l}}^{\hat{\mathrm{\alpha}}_{l}} \# \chi_{i_{l}, a_{l}}^{j_{l}}\right],
$$

where $\chi_{\hat{k}_{l}, \hat{\alpha}_{l}}^{\hat{p}_{l}}$ and $\chi_{i_{l}, a_{l}}^{j_{l}}$ are linked in $T_{1} \# \hat{T}_{1}=S_{2} \times S_{1}$ as indicated on Fig. 18 and $\mathscr{K}_{\hat{\mathscr{G}}}^{-}$ is depicted on the same figure.

Putting formulas (68) and (69) together and using (65) in the cases $g=0$ and $g=n=1$ shows that:

$$
\begin{gathered}
e^{2 \pi i g c \sigma_{1} / 8} \sum_{\mathscr{C}} \frac{Z\left[S_{3}, K_{+} \# \mathscr{G}_{\mathscr{C}}\right] Z\left[T_{g} \# \hat{T}_{g}, \mathscr{G}_{\hat{\mathscr{C}}} \# K_{-}\right]}{\left\langle T_{g}, \mathscr{G}_{\mathscr{C}} \mid T_{g}, \mathscr{G}_{\mathscr{C}}\right\rangle} \\
=Z\left[S_{3}, K_{n,+} \# K_{n,-}\right] \prod_{l=1}^{g} \frac{Z\left[S_{3}, \mathscr{L}_{l}\right]}{S_{0}^{j_{l}}}
\end{gathered}
$$
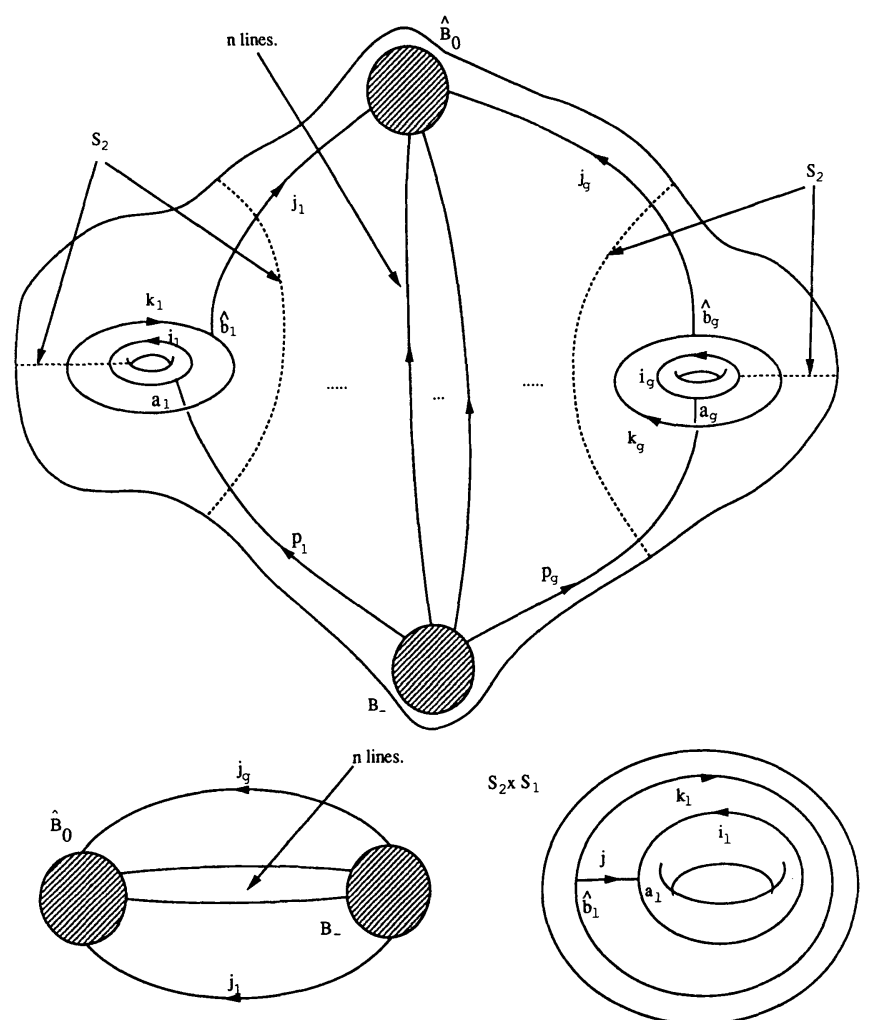

Fig. 18. $\left(\mathscr{G}_{\hat{\mathscr{E}}} \# K_{-}\right) \subset T_{g} \# \hat{T}_{g}$ and the auxiliary graphs used in the computation of $Z\left[T_{g} \# \hat{T}_{g}, \mathscr{G}_{\hat{q}} \# K_{-}\right]$ 


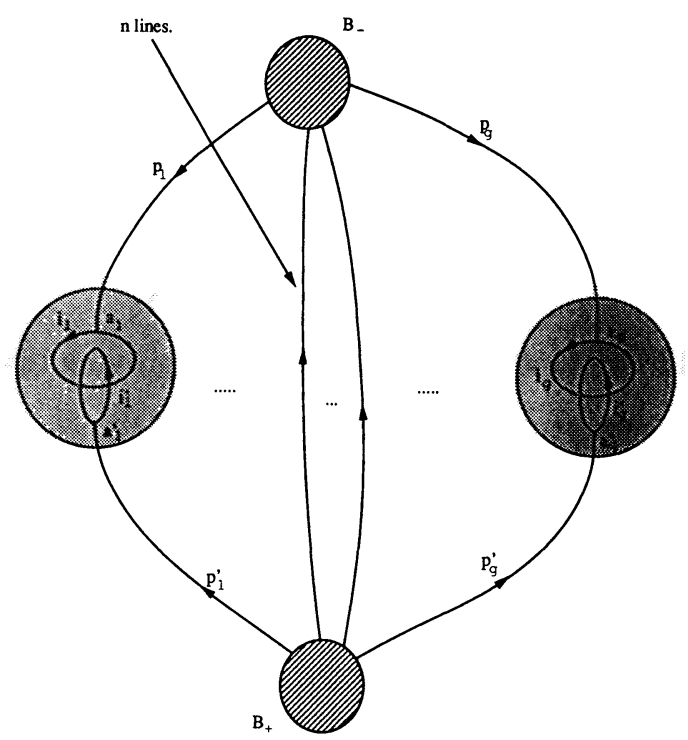

Fig. 19. Direct computation of $Z\left[S_{3}, K_{+} \# K_{-}\right]$by cutting along punctured spheres to isolate $K_{+, n} \# K_{-, n}$ from the $\mathscr{K}_{l}$ s. $K_{n,+} \# K_{n,-}$ is obtained by gluing $B_{ \pm}$using their external legs. In the same way, $\mathscr{L}_{l}$ is defined by closing the graph inside the $l$-th shadowed ball on our figure with a single line

where $K_{n, \pm}$ and $\mathscr{L}_{-}$are defined on Fig. 19 . We remark that $\sigma_{g}=g \sigma_{1}$ and cutting $\left[M_{+} \# M_{-}\right]$along suitable two-spheres which isolate $K_{ \pm}$'s bulbs $K_{n, \pm}$ from the $\mathscr{L}_{l}$ shows that the right-hand side of (70) is nothing but $Z\left[\bar{M}_{+} \# M_{-}\right]$. All this proves (65) when one line is running in each homotopically non-trivial loop of $M_{ \pm}$.

In the general case, more than one line runs through along the $(a)$ cycles in $S_{3} \backslash T_{g}$ or (b) cycles in $T_{g}$. The method is exactly the same as in the previous case and the interested reader will check it in detail: we can go back to the situation where only one line runs along each cycle, and we prove that:

Theorem 5. Projective partial functoriality holds in $S_{3}$ along any surface $\Sigma_{g, n}$.

B.2.5. Miscellanea. Let us prove that any line carrying a 0 index can be forgotten. We consider $K \subset S_{3}$ a trivalent framed colored graph a line of which carries the index 0 . Two possibilities are available:

- The line is a connected component of $K$. In this case, let us consider a tubular neighbourhood of this line $C_{0}$ in $S_{3}$ which does not intersect $K \backslash C_{0}$. We then cut along $\Sigma_{1,0}$ prove that one can delete $C_{0}: Z\left[S_{3}, K\right]=Z\left[S_{3}, K \backslash C_{0}\right]$.

- The line is not a connected component of $K$. At least one vertex connects it to the rest of $K$. Indeed, the basic situation is depicted on the following figure:

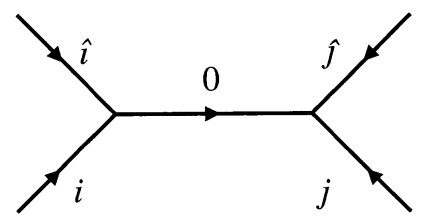


Of course, the $i, j$ indices can also be 0 . In this case, we use a four-punctured sphere to see that one can replace the preceding configuration of lines by:
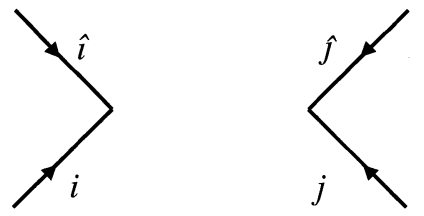

This is obtained using functoriality along $S_{2}$ and proving that:

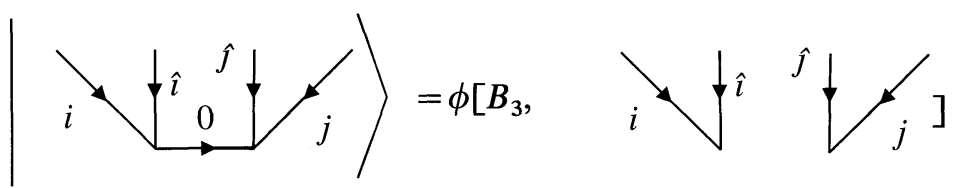

by explicit computation.

Henceforth, any line carrying the index 0 can be forgotten because we can first of all delete all isolated loops and then any line which relates two vertices. This step being completed, we may have created some isolated loops with index $0^{31}$. Then, we iterate this process and by induction over the number of line indices equal to 0 , it is possible to forget about all lines carrying index 0 !

\section{References}

1. Friedan, D., Shenker, S.: The analytic formulation of 2D CFT. Nucl. Phys. B 281, 509-545 (1987)

2. Vafa, C.: Conformal field theories and punctured surfaces Phys. Lett. B 199, 195 (1987); Towards classification of conformal field theories. Phys. Lett. B 206, 421-426 (1988)

3. Witten, E.: Quantum field theory and the Jones polynomial. Commun. Math. Phys. 121, 351-399 (1989)

4. Fröhlich, J.: 2D conformal field theory and 3D topology. Int. J. Mod. Phys. 20, 5321-5393 (1989)

5. Fröhlich, J., King, C.: Chern-Simons theory and the Jones polynomial. Commun. Math. Phys. 126, 167-199 (1989)

6. Verlinde, E., Verlinde, H.: Conformal field theory and geometric quantization. Preprint PUPT-90/1149 IASSNS-HEP-89/58

7. Dijkgraaf, R., Witten, E.: Topological Gauge theories and group cohomology. Commun. Math. Phys. 129, 393-429 (1990)

8. Moore, G., Seiberg, N.: Lectures on RCFT. Preprint RU-89-32 YCTP-P13-89

9. Crane, L.: Topology of 3-manifolds and conformal field theories. Preprint YCTP-P8-89 Kontsevitch, M.: Rational Conformal Field Theory and invariants of 3-dimensional manifolds. Preprint CPT-88/P.2189

10. Reshetikhin, N.Yu., Turaev, V.G.: Invariants of 3-manifolds via Link polynomials and quantum groups. Preprint 1990. Invent. Math. (to appear)

11. Kuperberg, G.: Involutory Hopf algebras and 3-manifold invariants. Preprint Berkeley 1990

12. Lickorish, W.B.R.: Invariants of three-manifolds from the combinatorics of the Jones polynomial. Preprint, Cambridge 1989

13. Moore, G., Seiberg, N.: Polynomial equations for rational conformal field theories. Phys. Lett. B 212, 451-460 (1988)

${ }^{31}$ For example, a loop with index 0 may be related to $K$ by a line with index 0 , we first delete the connecting line and this creates an isolated loop with index 0 
14. Moore, G., Seiberg, N.: Classical and quantum conformal field theory. Commun. Math. Phys. 123, 177-255 (1989)

15. Fröhlich, J.: Statistics of fields, the Yang-Baxter equation and the theory of knots and links. Non-perturbative quantum field theory. t'Hooft, G., Jaffe, A., Mack, G., Mitter, P.K., Stora, R. (eds.). New York: Plenum Press 1988

16. Rehren, K.-H., Schröer, B.: Einstein causality and Artin braids. Preprint FU-88-0439

17. Witten, E.: Gauge theories and integrable lattice models. Nucl. Phys. B 322, 629-697 (1989)

18. Frenkel, I.: Lecture at the metting on infinite dimensional symmetries in physics and mathematics. Paris, 1989

19. Segal, G.: The definition of conformal field theory. Proceeding of the Swansea Conference on Mathematical Physics

20. Atiyah, M.: Topological field theories. Publ. Math. IHES 68, 175-186 (1989)

21. Dijkgraaf, R.: A geometrical approach to 2 dimensional CFT. Thesis, Utrecht University (1989)

22. Witten, E.: Topological quantum field theory. Commun. Math. Phys. 117, 353-386 (1988)

23. Degiovanni, P.: TFT and RCFT. Preprint LPTENS 89/25

24. Reshetikhin, N.Yu., Turaev, V.G.: Ribbon graphs and their invariants derived from quantum groups. Commun. Math. Phys. 127, 1-26 (1990)

25. Lickorish, W.B.R.: Surgery on knots. Proc. A.M.S. 60, 296-298 (1977)

26. Kirby, R.: A calculus for framed links in $S_{3}$. Invent. Math. 45, 35-56 (1978)

27. Fenn, R., Rourke, C.: On Kirby's calculus of links. Topology 18, 1-15 (1979)

28. Motto, M.: Surfaces in 3-folds and maximal triads. Ph.D. Thesis at University of Texas, Austin

29. Birman, J.: Braids, links, and mapping class groups. Ann. Math. Studies, vol. 82. Princeton, NJ: Princeton University Press 1974

30. Witten, E.: The central charge in 3 dimensions. In: The Physics and Mathematics of Strings. Singapore: World Scientific Publ. Co. 1990

31. Atiyah, M.: On the framing of 3-manifolds. Topology 29, 1-7 (1990)

32. Harer, J.: The second homology group of the mapping class group of an orientable surface. Invent. Math. 72, 221-239 (1983)

33. Cappelli, A., Itzykson, C., Zuber, J.B.: The ADE classification of $A_{1}^{(1)}$ and minimal conformal field theories. Commun. Math. Phys. 113, 1-26 (1987)

34. Deligne, P., Milne, J.S.: Tannakian categories. Lecture Notes in Maths., vol. 900. Berlin, Heidelberg, New York: Springer 1982

35. Baulieu, L.: Lectures at Cargese 1989. Preprint PARIS-LPTHE/89-38

36. Baulieu, L., Singer, I.: Topological Yang-Mills symmetry. Nucl. Phys. B [Proc. Suppl.] 5, 12-19 (1988)

37. Altschüler, D., Coste, A.: Quasi-quantum groups, three-manifolds, and topological field theory. CERN-TH 6360/92, CPT-91/PE.263e4, ENSLAPP-A-360-91 
